\title{
Exposure to wild-type AAV drives distinct capsid immunity profiles in humans
}

\author{
Klaudia Kuranda, ${ }^{1}$ Priscilla Jean-Alphonse, ${ }^{1}$ Christian Leborgne, ${ }^{2}$ Romain Hardet, ${ }^{1}$ Fanny Collaud, ${ }^{2}$ Solenne Marmier, ${ }^{1}$ \\ Helena Costa Verdera, ${ }^{1}$ Giuseppe Ronzitti, ${ }^{2,3}$ Philippe Veron, ${ }^{2}$ and Federico Mingozzi ${ }^{1,2,3}$
}

IINSERM U974, Sorbonne Université, Paris, France. ²Genethon, Evry, France. ${ }^{3}$ INSERM S951, Université Evry, Université Paris Saclay, EPHE, Evry, France.

\begin{abstract}
Recombinant adeno-associated virus (AAV) vectors have been broadly adopted as a gene delivery tool in clinical trials, owing to their high efficiency of transduction of several host tissues and their low immunogenicity. However, a considerable proportion of the population is naturally exposed to the WT virus from which AAV vectors are derived, which leads to the acquisition of immunological memory that can directly determine the outcome of gene transfer. Here, we show that prior exposure to AAV drives distinct capsid immunity profiles in healthy subjects. In peripheral blood mononuclear cells (PBMCs) isolated from AAV-seropositive donors, recombinant AAV triggered TNF- $\alpha$ secretion in memory CD8 ${ }^{+} \mathrm{T}$ cells, $B$ cell differentiation into antibody-secreting cells, and anti-capsid antibody production. Conversely, PBMCs isolated from AAV-seronegative individuals appeared to carry a population of NK cells reactive to AAV. Further, we demonstrated that the AAV capsid activates IL-1 $\beta$ and IL- 6 cytokine secretion in monocyte-related dendritic cells (moDCs). IL-1 $\beta$ and IL- 6 blockade inhibited the anti-capsid humoral response in vitro and in vivo. These results provide insights into immune responses to AAV in humans, define a possible role for moDCs and NK cells in capsid immunity, and open new avenues for the modulation of vector immunogenicity.
\end{abstract}

\section{Introduction}

With several successful adeno-associated virus (AAV) vectorbased gene therapy clinical trials (1-4) and the recent approval by the FDA of a gene therapy for congenital blindness (5), the promise of a definitive cure for numerous genetic diseases is becoming a reality. Despite the dazzling progress thus far in the field, untoward immune responses to the AAV vector capsid remain the primary obstacle to the widespread adoption of this technology and, in some cases, to the achievement of long-lasting efficacy (6). Experience in the clinic with $\mathrm{AAV}$ vectors highlighted 3 main issues related to vector immunogenicity: (a) cellular immune responses against vector-transduced cells; (b) humoral immune responses occurring upon initial vector infusion; and (c) preexisting anticapsid antibodies able to neutralize the vector in individuals who were already exposed to WT AAV $(7,8)$.

The innate immune response to the AAV capsid in humans, compared with responses to adenoviral or lentiviral vectors, appears to be low and self-limited (9-12), although its impact on adaptive immune responses is poorly understood. In nonparenchymal liver cells, capsid particles are first sensed by TLR2 (13), which is known to act in a complex with CD14 (14). Then, probably via the NF- $\kappa$ B pathway, genes encoding for proinflammatory cyto-

Conflict of interest: FM and KK are inventors on a patent application describing the modulation of AAV vector immunogenicity (internal ref. B01806_PR2254). FM and KK are currently employees of Spark Therapeutics Inc., a company involved in the development and commercialization of AAV gene therapies.

License: Copyright 2018, American Society for Clinical Investigation.

Submitted: May 21, 2018; Accepted: September 11, 2018

Reference information: / Clin Invest. 2018;128(12):5267-5279.

https://doi.org/10.1172/JCl122372. kines such as IL-1 $\beta$ and IL- 6 are upregulated (13). In mice, it has been shown that TLR9 expressed in plasmacytoid dendritic cells (DCs) senses AAV vector genomes, facilitating the cross-presentation of capsid antigen to CD8 ${ }^{+} \mathrm{T}$ cells via conventional DCs (15).

The study of cell-mediated immune responses to the AAV capsid is problematic, as these responses are not easily induced in animal models (16-18), unless complex immunization protocols are used (19). In numerous studies in mice, dogs, and nonhuman primates, stable expression of a transgene was achieved, and the disease phenotype was corrected without any detectable activation of cytotoxic T cells (reviewed in ref. 20). In mice, activation of capsid-specific T cells depends on the TLR9 myeloid differentiation factor 88 (MyD88) pathway (21), however it always requires an additional proinflammatory stimulus, such as insertion of a foreign immunogenic peptide genetically engineered within the capsid structure (16), simultaneous expression of an immunogenic transgene (17), or administration of a proinflammatory cytokine (18). Capsid T cells are also elicited when the AAV capsid protein VP1, instead of being classically endocytosed by cells, is expressed by cells transduced with an adenoviral vector encoding for its sequence (17).

In contrast to animal models, human trials of AAV gene transfer showed in some instances liver toxicity imputable to a $\mathrm{T}$ cellmediated response against the capsid of the infused vector $(1,2,6$, 22-24), which was modulated using transient immunosuppressive treatment $(1-4,23,24)$. In current trials, $\mathrm{T}$ cell responses against therapeutic vectors are routinely monitored in peripheral blood mononuclear cells (PBMCs) using an IFN- $\gamma$ enzyme-linked immunospot (ELISPot) assay (1, 2, 6, 22-24). This technique barely detects AAV capsid-specific T cells in healthy individuals $(22,25$, 26), although the proportion of responding subjects is significantly 
increased after AAV gene transfer or prolonged in vitro restimulation of AAV-specific T cells $(22,25,27)$.

Unlike cellular responses (17, 22, 28, 29), humoral responses to AAV are robust in animal models. In mice, anti-AAV antibody production seems to depend on the B cell-intrinsic MyD88 signaling pathway $(21,30)$. Upon recombinant $\mathrm{AAV}$ infusion, mice and nonhuman primates develop high titers of anti-AAV-neutralizing antibodies (31-34), and it is also the case for humans (24, 35). Seroconversion does not affect the efficacy of gene transfer, however, it prevents vector readministration, as antibodies develop following AAV vector administration, like those elicited by natural AAV infection, persist over time and can efficiently neutralize the vector $(6,32,36,37)$.

To better understand and control immune responses directed against the AAV capsid, we analyzed multiple populations of immune cells present in the blood of healthy individuals and correlated the results with the AAV serology of the donors. We demonstrated that innate immune responses to the capsid in blood were mainly driven by $\mathrm{CD} 14^{+}$monocyte-related dendritic cells (moDCs), which secreted IL-1 $\beta$ and IL- 6 cytokines. In subjects previously exposed to WT AAV, these cytokines triggered the differentiation of capsid-specific memory B cells into antibodysecreting cells (ASCs) and anti-capsid antibody production. Both processes were inhibited in vitro and in vivo by IL- $1 \beta$-neutralizing antibodies. Capsid-specific TNF- $\alpha^{+}$memory CD $8^{+} \mathrm{T}$ cells were readily detectable in AAV-seropositive subjects, while the AAVseronegative individuals responded to the AAV capsid exclusively by the activation of discreet NK cell populations.

These insights into the innate and adaptive immune responses to the AAV capsid in humans are highly relevant to in vivo gene transfer, as they provide tools for immunomonitoring and define a novel strategy for the control of vector immunogenicity.

\section{Results}

$A A V$ capsid triggers $I L-1 \beta$ and $I L-6$ secretion in moDCs. It has been shown that in human liver nonparenchymal cells, the upregulation of IL-1 $\beta$ and IL- 6 expression in response to the AAV capsid depends on TLR2 and its accessory protein CD14 (13). Since blood monocytes express high levels of TLR2 and CD14 (38), we sought to determine whether a similar response to the AAV capsid occurs in $\mathrm{CD} 14^{+}$moDCs.

First, we measured IL-1 $\beta$ and IL-6 levels in PBMC cultures stimulated for 24 hours with AAV2 or influenza A (flu) pools of peptides. Compared with the unstimulated cultures, we found that AAV2 triggered a 4.8 -fold ( \pm 3.4 -fold) increase of IL- $1 \beta$ and an 8.5 -fold ( \pm 9.7 -fold) increase of the IL- 6 concentration in conditioned medium (Figure 1A). The increase in IL-1 $\beta$ and IL-6 secretion occurred in $72 \%$ of the tested samples (8 of 11) (Figure 1A) and was not related to the AAV2 serological status of the donors (Supplemental Figure 1; supplemental material available online with this article; https://doi.org/10.1172/JCI122372DS1). Further, to identify the cellular subset responsible for IL-1 $\beta$ and IL-6 secretion in the same experimental setting, we performed an intracellular cytokine staining (ICS) assay. We analyzed the adherent $\mathrm{CD}^{-}{ }^{-} \mathrm{CD} 19^{-}{ }^{-} \mathrm{LA}-\mathrm{DR}^{+} \mathrm{CD} 11 \mathrm{c}^{+}$cell fraction of PBMCs, which includes 3 subsets of myeloid DCs: CD11chi, CD11c ${ }^{\text {lo }}$, and CD $14^{+}$moDCs (Figure 1B) (39). IL-1 $\beta$ secretion in response to
AAV2 could be principally attributed to the moDC subset (Figure $1 \mathrm{C})$, since the average size of the IL- $1 \beta^{+}$subpopulation was the highest in moDCs (12\%), and IL-1 $\beta$ was most frequently detected in this subset of DCs (70\% of donors) (Figure 1C).

IL-6 secretion was less frequently detected in the ICS assay compared with the direct measurement in conditioned media. This could be due to the shorter cytokine accumulation time for the ICS assay (5 hours) compared with that for the Luminex assay (24 hours), or to the different measurement time windows (24-29 hours after restimulation in the ICS assay versus 0-24 hours in the Luminex assay). Nevertheless, increased IL-6 secretion in response to the AAV capsid was also detected by flow cytometry (Figure 1D) in 6 of 17 donors, and the moDCs were again the main cell population producing this cytokine (percentage of IL- $6^{+}$cells in each DC subset: CD11clo, $0.6 \% \pm 1.1 \%$; CD11 chi, $0.2 \% \pm 0.3 \%$; moDCs, $6.0 \% \pm 8.1 \%$ ) (Supplemental Figure 2).

The control flu pool of peptides did not trigger significant changes in IL-1 $\beta$ or IL- 6 secretion (Figure 1, A, C, and D), despite the fact that several subjects had antibodies against both AAV and flu (Supplemental Table 1 and Supplemental Figure 3). Conversely, when we measured the maturation state of DCs in the same conditions, we found that flu, but not AAV2, triggered CD86 upregulation in the $3 \mathrm{DC}$ subsets (Figure 1E). These results suggest that AAV and flu interact differently with the host immune system.

PBMCs were also restimulated in parallel with the AAV2 pool of peptides or with empty AAV2 capsid particles. We then performed an ICS assay, which confirmed that intact capsid particles elicited similar responses to those observed upon restimulation with the pool of capsid peptides (Figure 1F). Collectively, these data identify moDCs as the main innate responders to the AAV capsid in human peripheral blood.

High-dimensional analysis of the immune responses to $A A V$ in PBMCs from healthy donors highlights distinct populations of capsidreactive immune cells. To identify cellular subsets involved in the immune response to the AAV2 capsid, we stimulated PBMCs isolated from 4 healthy donors with empty AAV2 viral particles for 48 hours in vitro, followed by cytometry by time-of-flight (CyTOF) analysis. We measured concomitant cytokine secretion (TNF- $\alpha$, IFN- $\gamma$, IL-2, IL-5, IL-10, and IL-17a), activation (CD25, HLA-DR), and recent activation and exhaustion (PD-1, CD57) markers in the 11 cell subsets shown in Figure 2A. In agreement with previously published observations $(22,26,40)$, we found that AAV2 capsid triggered a response in $\mathrm{CD}^{+} \mathrm{T}$ cells (Figure 2B). These cells showed increased TNF- $\alpha$ and granzyme B secretion and signs of recent activation/exhaustion, indicated by $\mathrm{PD}-1$ upregulation (41). Multiparametric analysis permitted the precise characterization of this $\mathrm{CD}^{+} \mathrm{T}$ cell subset as that of effector memory (EM) cells $\left(\mathrm{CD}^{2} 5^{+} \mathrm{CD}^{+} \mathrm{CD}^{+} \mathrm{CD}^{+} 5 \mathrm{RO}^{-} \mathrm{CD} 45 \mathrm{RA}^{-}\right)$. IFN- $\gamma$ secretion was detectable neither in $\mathrm{CD}^{+}$nor in $\mathrm{CD}^{+} \mathrm{T}$ cells, while its robust secretion was observed in the positive control, as represented by PBMCs treated with PMA and ionomycin (Supplemental Figure 5). Importantly, in 3 of the 4 donors tested, AAV capsid triggered the secretion of TNF- $\alpha$ and IFN- $\gamma$ as well as the upregulation of HLA-DR in NK cells $\left(\mathrm{CD} 45^{+} \mathrm{CD}^{-} \mathrm{CD}^{-} 9^{-} \mathrm{CD} 16^{+}\right)$(Figure 2B), indicating the activation of this immune cell population (42). Only 2 of 11 immune cell populations tested responded to the capsid antigen stimulation, confirming the overall low immunogenicity of AAVs. 
A
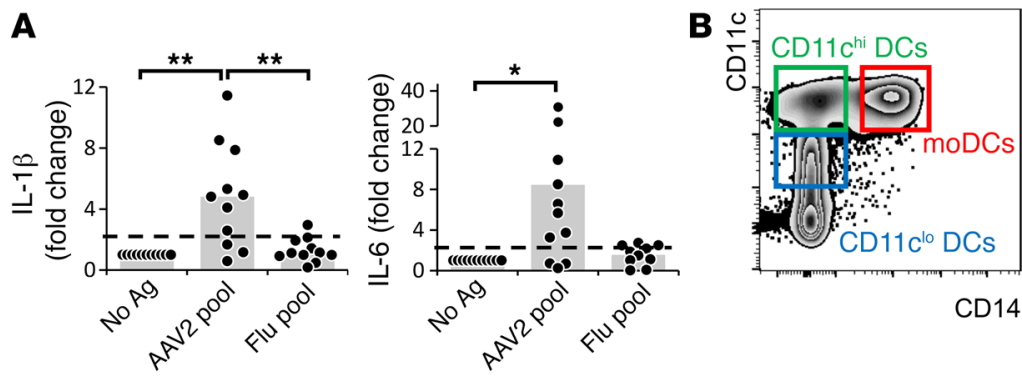

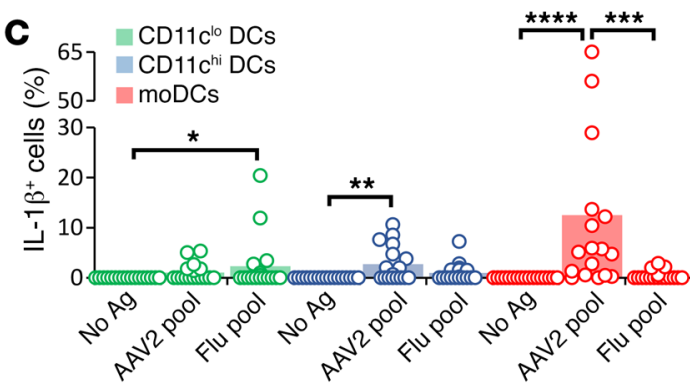

D

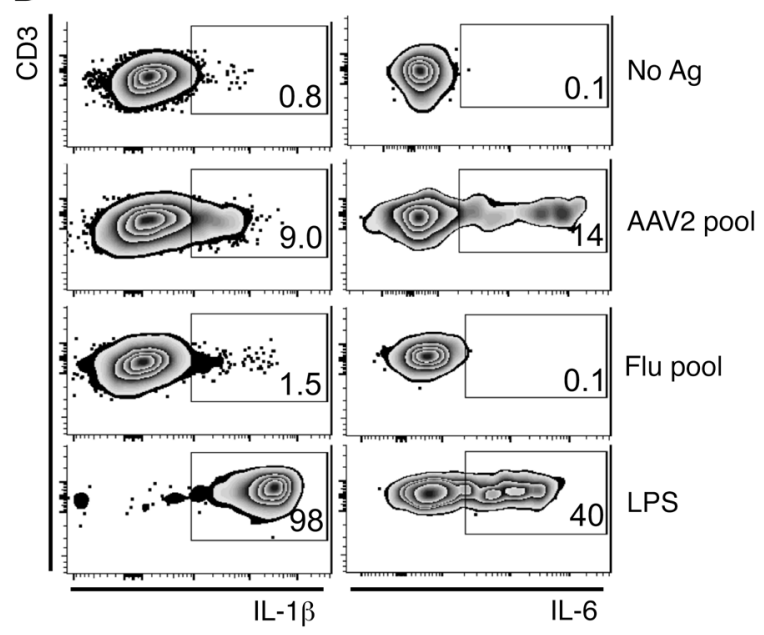

E

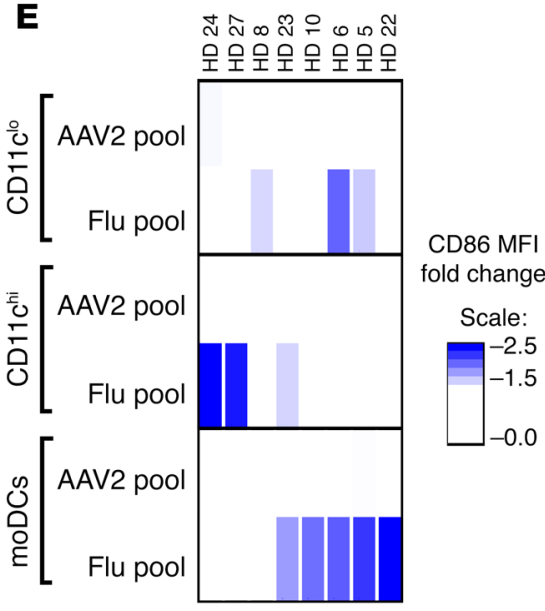

$\mathbf{F}$

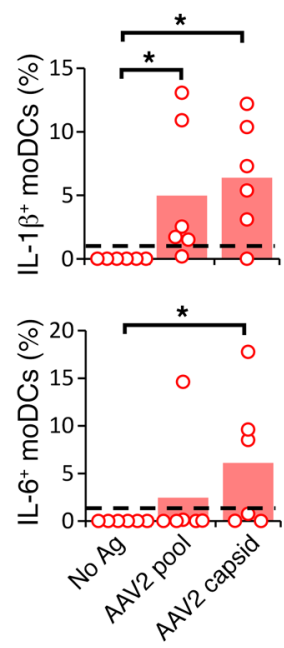

Figure 1. AAV capsid triggers IL-1 $\beta$ and IL-6 secretion in human moDCs. (A) Fold change of IL-1 $\beta$ and IL-6 concentration in antigen-treated PBMC cultures versus controls without antigen (No Ag), measured by Luminex 24 hours after restimulation. (B) Representative flow cytometric plot showing the phenotype of DC populations analyzed in C, D, E, and F. Adherent cells were gated on single, live CD3-CD19-HLA-DR ${ }^{+}$cells. (C) Percentage of cytokine-positive cells in a given DC subset measured by ICS assay 24 hours after restimulation. (D) Representative flow cytometric plots showing IL-1 $\beta$ or IL-6 staining in moDCs and the percentage of positive cells. Stimulation with LPS was used as a positive control. (E) Heatmap representing the fold changes of mean fluorescence intensity (MFI) for CD86 staining in the indicated DC populations, measured by flow cytometry 24 hours after restimulation. HD, healthy donor. (F) Percentage of cytokine-positive cells in moDC subsets measured by ICS assay 24 hours after restimulation with either peptides or whole AAV2 capsid. In $\mathbf{A}, \mathbf{C}$, and $\mathbf{F}$, histograms show the mean, and symbols represent individual sample values. Dashed lines represent the positive cutoff. ${ }^{*} P<0.05$, ${ }^{* *} P<0.01,{ }^{* *} P<0.001$, and ${ }^{* * *} P<0.0001$, by nonparametric Kruskal-Wallis 1-way ANOVA with Dunn's multiple comparisons test.

Interestingly, NK cells appeared to be involved in immune recognition of the AAV2 capsid.

Identification of capsid-specific IFN- $\gamma^{+} C D 16^{\text {bright }} C D 56^{\text {dim }} \mathrm{NK}$ cells in $A A V$-seronegative individuals. Since CyTOF analysis pointed to the activation of NK cells in response to the AAV2 capsid, we sought to further characterize this immune cell subset. To facilitate internalization, processing, and presentation of the AAV capsid by antigen-presenting cells (APCs), we used a peptide pool spanning the full sequence of the AAV2 capsid protein VP1 for PBMC stimulation. An ICS assay, followed by conventional flow cytometry, confirmed the CyTOF results and showed that both IFN- $\gamma$ and TNF- $\alpha$ were secreted by a small subset of CD16 ${ }^{\text {bright }}$ CD $56^{\mathrm{dim}} \mathrm{NK}$ cells (Figure $3 \mathrm{~A}$ ). On average, IFN- $\gamma^{+}$cells constituted $0.2 \% \pm 0.05 \%$ and TNF- $\alpha^{+}$cells $0.3 \% \pm 0.16 \%$ of the total CD $16^{\text {bright }}$ CD $56^{\mathrm{dim}} \mathrm{NK}$ cell population. Secretion of IFN- $\gamma$ in conditioned media from PBMC cultures stimulated with the AAV2 peptide pool was confirmed by an independent assay using Luminex technology (positive in 57\% of donors, Figure 3B). Capsid-responding NK cells did not seem to be cytotoxic, as suggested by the unchanged levels of granzyme
B (Figure 2B) and CD107a (Figure 3C), and secreted IFN- $\gamma$ and TNF- $\alpha$ transiently, with a peak occurring 24 hours after antigen stimulation (Figure 3C). Further, to verify whether the observed NK cell activation was specific to the AAV, we stimulated PBMCs in parallel with AAV serotypes 5 and 8, which share, respectively, $57 \%$ and $83 \%$ homology with the AAV2 VP1 sequence (43), or with different peptide pools derived from other viruses common in the cell population, such as flu, EBV, or CMV. This experiment showed that IFN- $\gamma$ secretion was triggered in $\mathrm{CD} 16^{\text {bright }} \mathrm{CD} 56^{\mathrm{dim}} \mathrm{NK}$ cells only by the peptide pools of AAV serotypes 2,5 , and 8 but not by other viral antigens, demonstrating that reactivity was specific to the AAV capsid (Figure 3D). Overall, in the examined cohort of healthy subjects $(n=17)$, we found that $53 \%$ of the donors had AAV2-induced IFN- $\gamma$ secretion from NK cells, $66 \%$ of which secreted TNF- $\alpha$ simultaneously (Figure $3 \mathrm{E}$ ).

While seropositivity is a general indication of previous exposure to a virus, we verified whether NK activation could be related to the AAV serological status of the donors tested. We measured anti-capsid antibody titers in sera from donors, focusing 

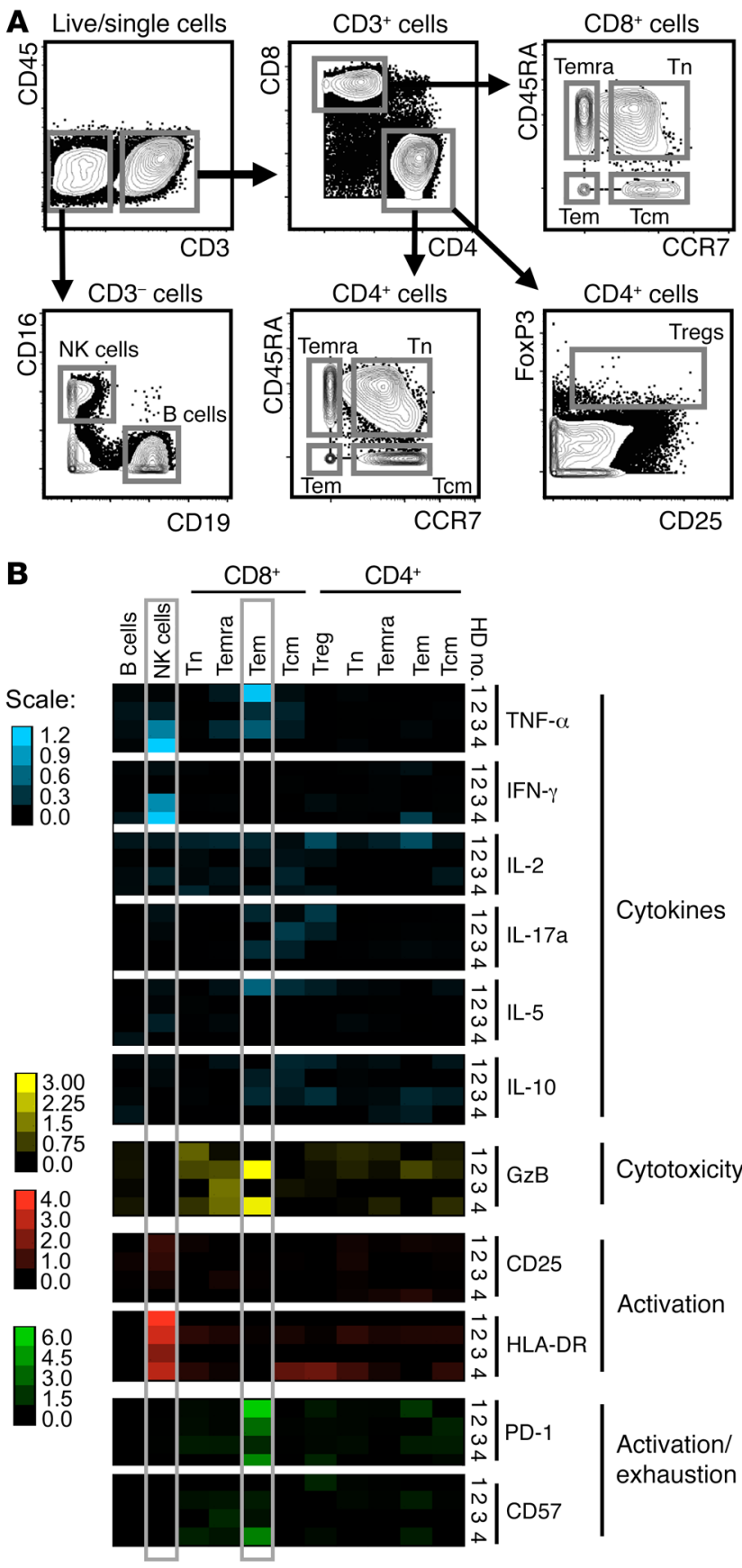

on AAV2, as it naturally infects humans and is therefore the most seroprevalent of all AAV serotypes (44). Strikingly, capsid-specific IFN- $\gamma^{+} \mathrm{CD} 16^{\text {bright }} \mathrm{CD} 56^{\text {dim }} \mathrm{NK}$ cells were detected only in PBMCs isolated from seronegative donors who were probably not exposed to the WT virus (Figure 3F).

Together, these results suggest that seronegative individuals react to the AAV2 capsid with the transient activation of NK cells.

Identification of capsid-specific TNF- $\alpha^{+} C D 8^{+} T$ cells in AAV2seropositive individuals. TNF- $\alpha$ appeared to be the main cytokine secreted by $\mathrm{CD} 8^{+} \mathrm{T}$ cells in response to the AAV capsid (Figure $4 \mathrm{~A})$. To confirm this result in a larger number of healthy individuals, we isolated PBMCs from 11 donors and analyzed them using conventional flow cytometry. PBMC cultures were stimu-
Figure 2. CyTOF high-dimensional analysis of response to the AAV capsid in immune cell populations present in blood. (A) CyTOF plots showing the cellular subsets analyzed. Tcm, central memory T cells; Tem, effector memory T cells; Temra, effector memory T cells reexpressing CD45RA; $\mathrm{Tn}$, naive T cells. Preliminary gating of live and single cells is shown in Supplemental Figure 4. (B) Heatmap representing the percentage of cells positive for a given marker in each cellular subset. The background, as measured in the control cultures without antigen, was subtracted. Total PBMCs obtained from 4 healthy donors were analyzed by CYTOF 48 hours after restimulation with the empty AAV2 capsid particles.

lated with AAV2 or flu pools of peptides for 6 hours, and then an ICS assay was performed to measure the levels of TNF- $\alpha$, IFN- $\gamma$, IL-2, and CD107a. This experiment showed a significant $(\geq 0.1 \%$ positive cutoff) increase in TNF- $\alpha$ secretion in $54 \%$ of the samples tested (Figure 4A). Furthermore, in order to analyze a higher number of AAV2-specific CD8 ${ }^{+} \mathrm{T}$ cells and possibly increase the sensitivity of the ICS assay, we expanded antigen-specific T cells for 2 weeks in vitro. PBMCs were incubated with AAV2 and EBV peptide pools or AAV-1 and AAV-2 empty capsid particles. After expansion, we restimulated the cells with autologous DCs loaded with the cognate antigens. Background levels were measured in cultures in which expanded $\mathrm{T}$ cells were mixed with autologous DCs not exposed to antigens. While the percentage of capsidspecific TNF- $\alpha^{+} \mathrm{CD}^{+} \mathrm{T}$ cells increased on average from $0.16 \%$ before expansion to $1.4 \%$ after expansion ( $~ 8$ fold) and, additionally, the numbers of $\mathrm{IL}^{-} 2^{+}$and $\mathrm{CD} 107 \mathrm{a}^{+}$cells increased in a few donors, the expansion did not allow the detection of IFN- $\gamma^{+}$cells (Figure 4B). Further, we addressed the same question using MHC class I dextramers (DMrs) to more precisely identify antigen-specific $\mathrm{T}$ cell populations. We used phycoerythrin-conjugated (PE-conjugated) HLA- $B^{*} 0702$ dextramers loaded with the $B^{*} 0702$-restricted AAV2 immunodominant peptide VP1 ${ }_{372-380}(6,22,25)$ or the control EBV peptide EBNA $3 \mathrm{~A}_{247-255^{\circ}}$. PBMCs from an HLA-B ${ }^{*} 0702$ donor were expanded for 2 weeks (Supplemental Figure 6) and restimulated with an HLA- $B^{*} 0702$ cell line pulsed with a relevant antigen or an irrelevant one used as a negative control. This experiment confirmed that expanded capsid-specific $\mathrm{CD}^{+} \mathrm{T}$ cells were able to secrete TNF- $\alpha$ and IL-2 and degranulate in the presence of target cells, but did not secrete IFN- $\gamma$ (Figure 4C). Furthermore, unexpanded PBMCs from healthy donors were analyzed by a dual-color IFN- $\gamma /$ TNF- $\alpha$ FluoroSpot assay, in which secreted cytokines were captured for 24 hours on an antibody-coated polyvinyl membrane. This experiment confirmed the lack of IFN- $\gamma^{+}$secretion in response to the AAV capsid (Figure 4D). Altogether, these results demonstrate that, when tested ex vivo, TNF- $\alpha$ secretion is the main trait of capsid-specific $\mathrm{CD} 8^{+} \mathrm{T}$ cells isolated from healthy donors.

Finally, we verified whether the ex vivo detection of capsidspecific $\mathrm{TNF}-\alpha^{+} \mathrm{CD} 8^{+} \mathrm{T}$ cells was related to the AAV2 serological status of donors. We detected capsid-specific TNF- $\alpha^{+} \mathrm{CD} 8^{+} \mathrm{T}$ cells only in seropositive donor PBMCs (Figure 4E), which thus lacked capsid-specific IFN- $\gamma^{+} \mathrm{NK}$ cells (Figure $4 \mathrm{~F}$ ). These results, combined with the results of the CyTOF assay, suggest that capsid-specific memory $\mathrm{CD} 8^{+} \mathrm{T}$ cell responses are characterized by TNF- $\alpha$ secretion and can be found in seropositive, "AAV-experienced" individuals.

$A A V$ capsid triggers limited $C D 4^{+} T$ cell activation in a small proportion of donors. The ICS assays performed ex vivo or after 1 cycle 
A Gated on CD16 bright CD56 dim cells:

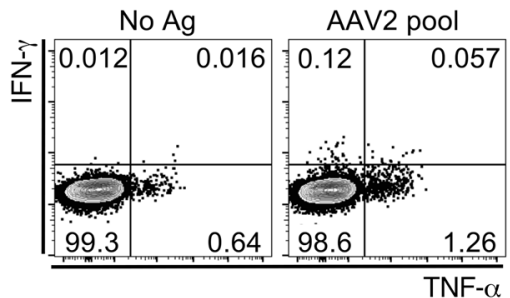

B
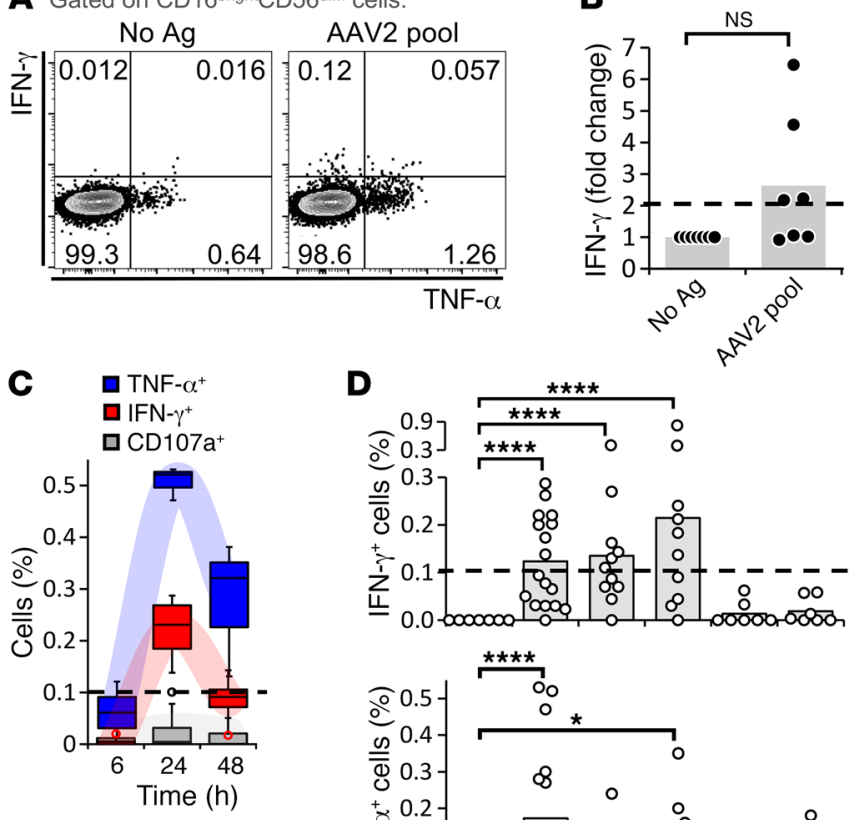

D
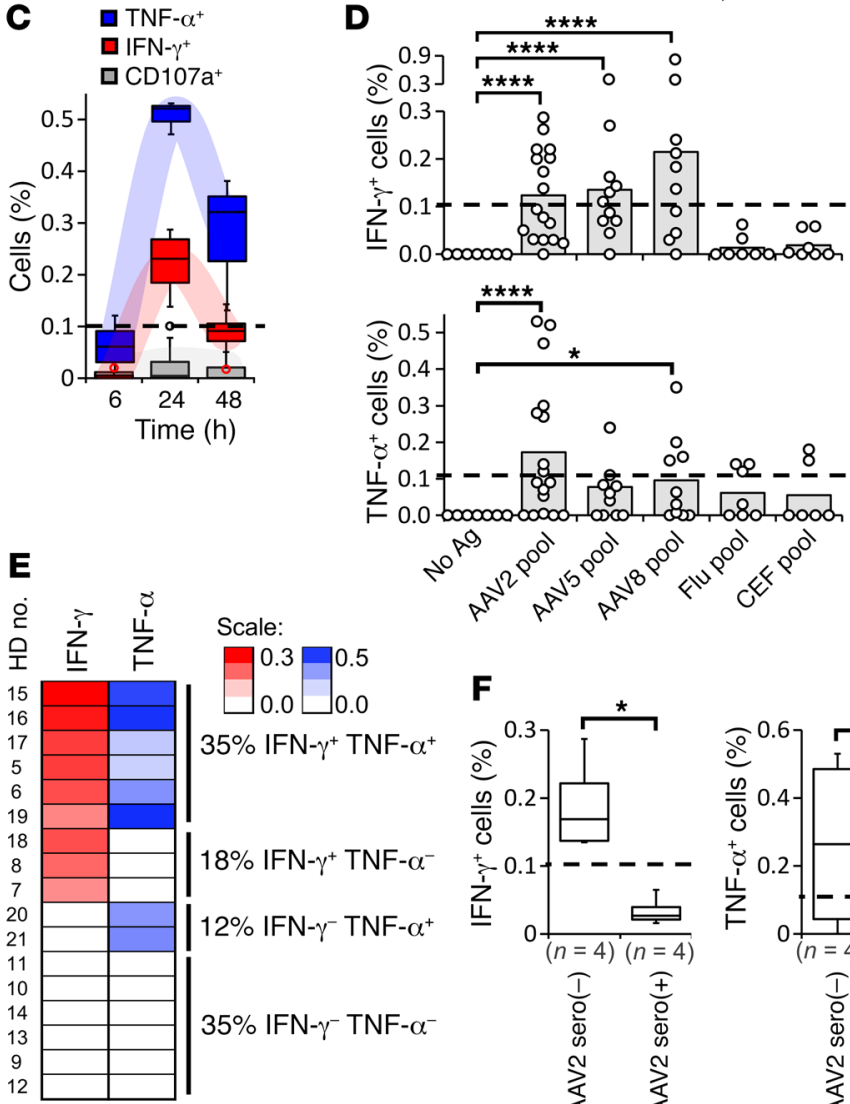

$\mathbf{F}$
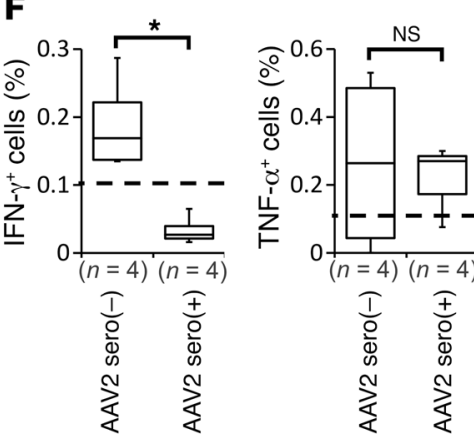

Figure 3. Identification of capsid-specific IFN- $\gamma^{+}$CD16 ${ }^{\text {bright }}$ CD56 $6^{\text {dim }}$ NK cells in AAV-seronegative individuals. (A) Flow cytometric plots showing IFN- $\gamma$ and TNF- $\alpha$ staining of NK cells, 24 hours after stimulation of PBMCs with the AAV2 pool of peptides or in control cultures without antigen. (B) Fold change of IFN- $\gamma$ concentration in the culture medium of PBMCs, measured by Luminex assay 24 hours after stimulation with the AAV2 pool of peptides. (C) Kinetics of IFN- $\gamma$ and TNF- $\alpha$ secretion and CD107a levels in CD16 bright CD56 ${ }^{\text {dim }}$ NK cells 6,24 , and 48 hours after stimulation with the AAV2 pool of peptides $(n=4)$. Box plots show the median \pm SD. (D) Percentage of cytokine-positive CD16 bright CD56 dim NK cells 24 hours after restimulation with the indicated antigens. (E) Heatmap representing the percentage of IFN- $\gamma^{+}$or TNF- $\alpha^{+} \mathrm{CD} 16^{\text {bright }}$ CD56 dim NK cells 24 hours after stimulation with the AAV2 pool of peptides. The percentage of healthy donors in each category is shown. (F) Percentage of IFN- $\gamma^{+}$or TNF- $\alpha^{+}$CD16 bright $C D 56^{\text {dim NK }}$ cells 24 hours after stimulation with the AAV2 pool of peptides measured in AAV2-seronegative or -seropositive donors. Box plots show the median \pm SD. In $\mathbf{B}$ and $\mathbf{D}$, the histograms show the mean, and the symbols represent individual sample values. Dashed lines indicate the cutoff for positivity. ${ }^{*} P<0.05$ and ${ }^{* * *} P<0.0001$, by Wilcoxon signed-rank test $(\mathbf{A})$, nonparametric Kruskal-Wallis 1-way ANOVA with Dunn's multiple comparisons test (D), and nonparametric Mann-Whitney $U$ test (F). of expansion permitted the concomitant characterization of $\mathrm{CD}^{+}$ and $\mathrm{CD} 4^{+} \mathrm{T}$ cells. The $\mathrm{CD} 4^{+} \mathrm{T}$ cell response to the AAV2 pool of peptides ex vivo was also limited to the TNF- $\alpha$ secretion, yet the percentage of responding donors was much lower than that for $\mathrm{CD}^{+} \mathrm{T}$ cell responses (27\% of donors) (Figure $5 \mathrm{~A}$ ). After a short expansion period in vitro, we could also detect sporadic secretion of IL-2, degranulation (CD107a), and modest IFN- $\gamma$ secretion in response to the AAV capsid particles but not the peptide pool (Figure $5 \mathrm{~B})$. We also used Luminex to detect "helper" cytokines that can be produced by CD $4^{+} \mathrm{T}$ cells, i.e., IL- 2 and IL- 4 , and that can be important for antibody production. While this method permitted better detection of IL-2 secretion in PBMCs stimulated with the flu pool of peptides, only 3 of 11 tested samples responded to stimulation with the AAV2 peptides (Figure 5C). Interestingly, 6 of 17 samples tested displayed increased IL-4 levels of at least 2-fold over background in response to AAV2 $(0.6 \mathrm{pg} / \mathrm{ml} \pm 0.2 \mathrm{con}$ trol cultures, $2.3 \mathrm{pg} / \mathrm{ml} \pm 1.4$ after stimulation with the AAV2 pool of peptides) (Figure $5 \mathrm{C}$ ). Detection of $\mathrm{TNF}-\alpha^{+} \mathrm{CD} 4^{+} \mathrm{T}$ cells or the increase in IL-4 levels in AAV-treated cultures was not related to the AAV2 seropositivity status of the corresponding donors (Supplemental Figures 7 and 8).

Overall, these results indicate that $\mathrm{CD} 4^{+} \mathrm{T}$ cell responses to the AAV capsid in healthy donors are limited when compared with $\mathrm{CD} 8^{+} \mathrm{T}$ cell responses.

AAV capsid triggers $I L-1 \beta$ - and IL-6-dependent B cell differentiation in vitro. The AAV capsid is known to induce humoral responses in humans and in animal models $(24,31-35)$. To study these responses in vitro, we stimulated human PBMC cultures with pools of AAV2 or flu peptides and performed immunophenotyping of B cells 7 days later using flow cytometry. Treatment with both antigen pools increased B cell differentiation, which was illustrated by an increase in the number of ASCs $\left(\mathrm{CD}^{-} \mathrm{CD}^{-} 9^{+} \mathrm{IgD}^{-}\right.$ $\mathrm{CD} 24^{-} \mathrm{CD} 27^{+} \mathrm{CD} 38^{++}$) in cell cultures (Figure 6A). Comparison of these results with the serum levels of the anti-AAV2 antibodies in the corresponding donors showed a 7-fold higher frequency of AAV2-specific ASCs in the seropositive donors compared with frequencies in seronegative donors (Figure 6B). Given these results and the fact that seronegative individuals probably carry only 

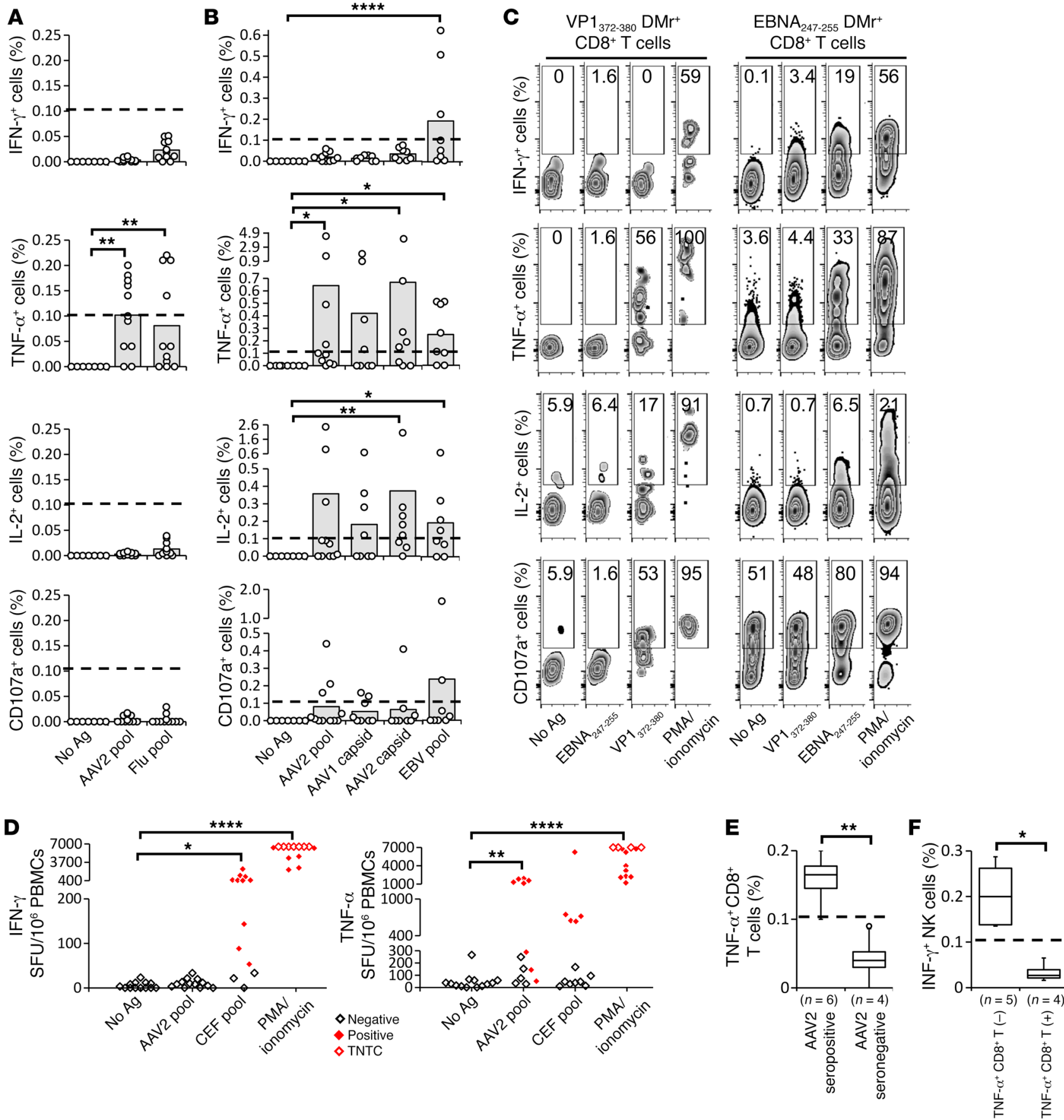

Figure 4. Identification of capsid-specific TNF- $\alpha^{+}$CD8 $8^{+} \mathbf{T}$ cells in AAV-seropositive individuals. (A and B) The percentage of positive cells for a given marker in CD8 ${ }^{+}$T cells was measured by ICS assay. The background, as measured in the control cultures without antigen, was subtracted. Histograms show the mean, and open symbols represent individual sample values. Dashed lines indicate the positive cutoff. (A) The percentage of positive cells was measured ex vivo 6 hours after restimulation of PBMCs. (B) The percentage of positive cells was measured after 1 cycle of expansion in vitro with the indicated antigens, and the recall was measured with antigen-loaded autologous DCs. (C) The percentage of positive cells for a given marker in DMr+CD8 ${ }^{+} T$ cells was measured after 1 cycle of expansion in vitro with the AAV2 peptide VP1 ${ }_{372-380}$ or with the control EBV peptide EBNA ${ }_{247-255}$, and the recall was measured with an HLA-matched antigen-pulsed cell line. (D) Cytokine secretion was measured by ELISpot, expressed as the number of SFU per $10^{6}$ PBMCs. Cells were stimulated for 24 hours with the indicated antigens. Black open symbols indicate a negative response; solid red symbols indicate a positive response; red open symbols indicate a positive response with SFU too numerous to count (TNTC). (E) The percentage of AAV2 capsid-specific TNF- $\alpha^{+} C D 8^{+}{ }^{2}$ cells was measured ex vivo in PBMCs from seropositive or seronegative donors. (F) Percentage of AAV2 capsid-specific IFN- $\gamma^{+}$NK cells in PBMCs comprising or not the capsid-specific TNF- $\alpha^{+} C D 8^{+} T$ cells. In $\mathbf{E}$ and $\mathbf{F}$, box plots show the median $\pm \mathrm{SD}$. ${ }^{*} P<0.05$, ${ }^{*} P<0.01$, and ${ }^{* * * *} P<0.0001$, by nonparametric Kruskal-Wallis 1-way ANOVA with Dunn's multiple comparisons test (A and $\mathbf{B}$ ) and nonparametric Mann-Whitney $U$ test (E and $\mathbf{F})$. $P$ values in $\mathbf{D}$ were determined by 2-tailed Student's $t$ test (80). 
A
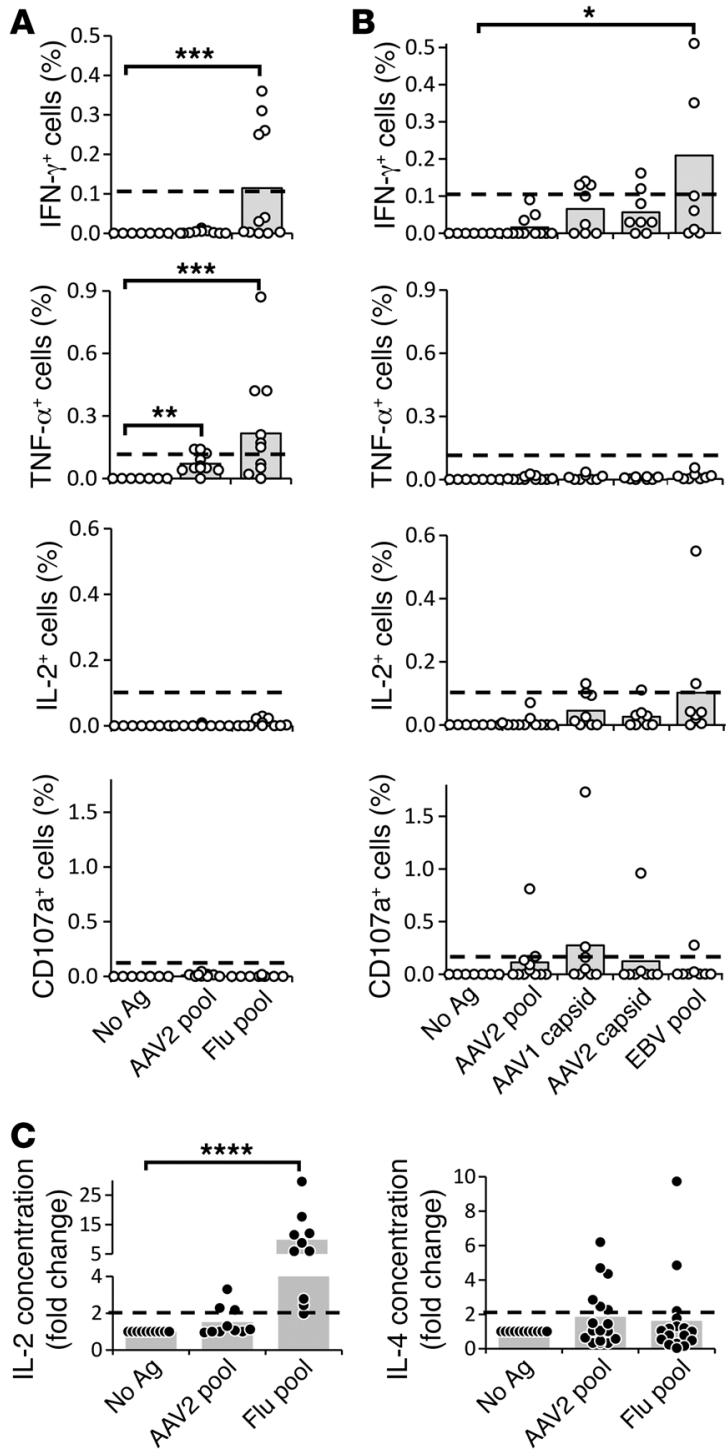

naive $B$ cells, it is plausible that in this experimental setting, the AAV capsid could stimulate only AAV2-specific memory B cells. In agreement with our results on T cell and NK cell responses, AAV2 triggered B cell differentiation in AAV2-seropositive donors in whom we also identified capsid-specific TNF- $\alpha^{+} \mathrm{CD} 8^{+} \mathrm{T}$ cells (Figure 6C), but this was not observed in the AAV2-seronegative donors who carried capsid-specific IFN- $\gamma^{+} \mathrm{CD} 16^{\text {bright }} \mathrm{CD} 56^{\text {dim }}$ NK cells (Figure 6D).

Further, we stimulated PBMCs from 3 AAV2-seropositive donors using empty AAV2 particles and measured anti-AAV2 IgM levels secreted into the culture medium. We focused on IgM Igs, as they are the first Igs produced in response to infection, can be rapidly secreted, even prior to isotype switching, and do not require $\mathrm{T}$ cell help. The levels of anti-AAV2 IgM increased on average by 1.5-fold when compared with basal levels in unstimulated cultures (Figure 6E), showing that increased AAV-specific ASC frequencies coincided with higher anti-AAV2 antibody levels. We observed no significant change in anti-AAV2 IgG secretion levels in these donors in response to capsid restimulation (Supplemental Figure 9), reflecting the prior exposure to AAV.
Figure 5. AAV capsid triggers limited $\mathrm{CD4}^{+} \mathrm{T}$ cell activation in a small proportion of donors. (A and $\mathbf{B}$ ) The percentage of positive cells for a given marker in the subset of $C D 4^{+} T$ cells was measured by ICS assay. The background, as measured in the control cultures without antigen, was subtracted. (A) The percentage of positive cells was measured ex vivo, 6 hours after restimulation of PBMCs. (B) The percentage of positive cells was measured after 1 cycle of expansion in vitro, and the recall was measured with the antigen-loaded autologous DCs. (C) Fold change of the IL-2 or IL-4 concentration in the culture medium of PBMCs, measured by Luminex assay 24 hours after stimulation with the AAV2 pool of peptides. The histograms in A-C indicate the mean, the symbols represent individual sample values, and the dashed lines represent the threshold of positivity. ${ }^{*} P<0.05$, ${ }^{* *} P<0.01,{ }^{* *} P<0.001$, and ${ }^{* * * *} P<0.0001$, by nonparametric KruskalWallis 1-way ANOVA with Dunn's multiple comparisons test.

Since we demonstrated that the AAV2 capsid triggered IL-6 and IL-1 $\beta$ secretion from moDCs in vitro, and since both cytokines are known to enhance the differentiation of B cells and antibody production (45-47), we tested whether neutralization of these cytokines could have any effect on B cell differentiation. We demonstrated that the addition of IL-1 $\beta$ - or IL-6-neutralizing antibodies to the cell cultures blocked AAV capsid-induced, but not flu-induced, B cell differentiation (Figure 6F and Supplemental Figure 10). Accordingly, in these conditions, we detected a reduction of secreted anti-AAV2 IgM (Figure 6G). Once again, these results highlighted divergent immune responses to AAV when compared with responses to other viral antigens.

These results support a model in which the AAV2 capsid induces B cell differentiation into ASCs and anti-AAV antibody production in vitro. This process is dependent on IL- $1 \beta$ and IL- 6 secretion and can be inhibited by antibodies that neutralize these cytokines.

$I L-1 \beta$-neutralizing antibodies control humoral responses to the $A A V$ capsid in vivo. Unlike cell-mediated immune responses (17, $22,28,29$ ), humoral responses to AAV are robustly elicited in small and large animal models upon vector administration (36, $48)$. To test whether the blocking of IL-1 $\beta$ and IL-6 in vivo could have an effect on anti-AAV antibody formation similar to that observed in vitro with human cells, we performed an AAV-based gene transfer experiment in immunocompetent C56BL/6 mice. We used the AAV8 serotype, which is highly homologous to AAV2 (49) but has a greater capacity to transduce murine hepatocytes (50). Both serotypes are also known to elicit similar immune responses in vitro and in vivo $(6,13,23,24)$ in terms of antibody production (30). Three groups of mice were injected i.v. with an AAV8 vector encoding for human factor IX (hF.IX). A group of animals received in parallel anti-IL-1 $\beta$-neutralizing antibodies, a second group received anti-IL-6-neutralizing antibodies, and a third group received an isotype control (Figure 6H). Three weeks after vector injection, anti-AAV8 IgG antibody levels were tested in blood and showed a significant decrease in antibody levels in the group treated with anti-IL-1 $\beta$-neutralizing antibodies (Figure $6 \mathrm{I}$ ). At the same time, vector genome copy numbers (VGCNs) in the liver confirmed that all groups of mice received equal doses of the vector and that the transduction of hepatocytes was successful in all groups (Figure 6J).

These results demonstrate that IL-1 $\beta$-neutralizing antibodies can be a potentially useful tool to reduce capsid immunogenicity in AAV vector-mediated gene transfer. 
A

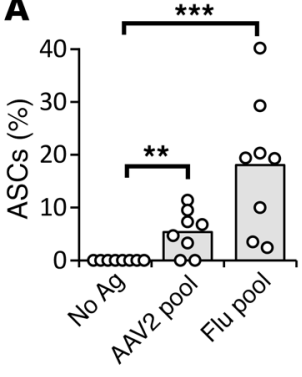

B

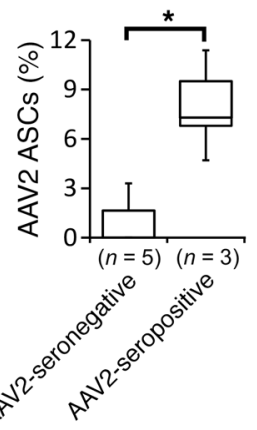

$\mathbf{F}$

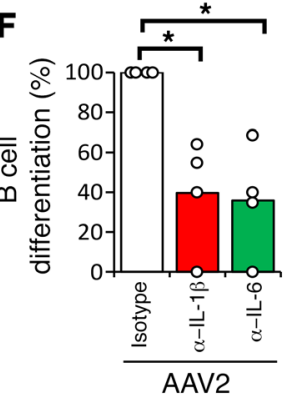

C

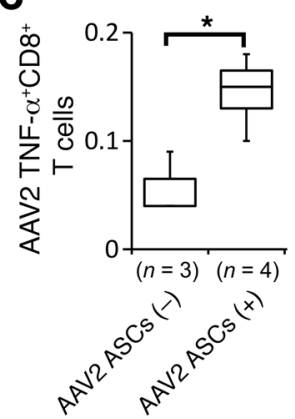

D

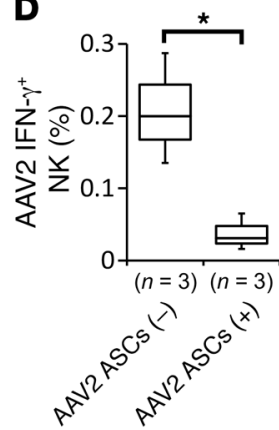

E

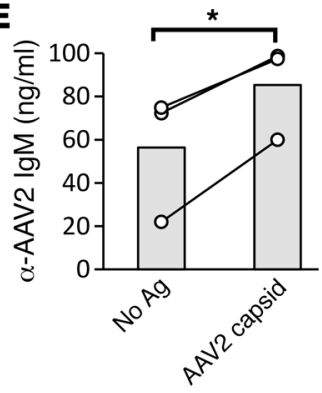

H

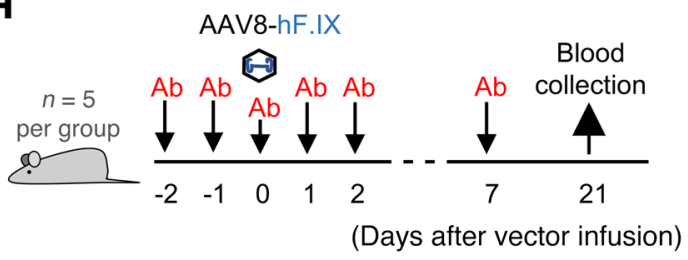

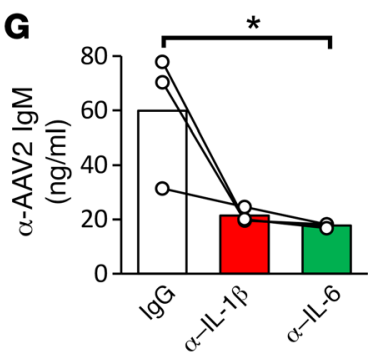

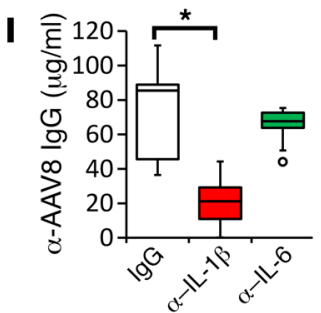

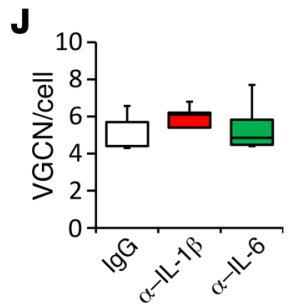

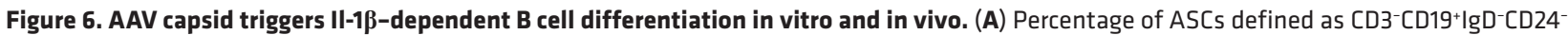
CD27 ${ }^{+} \mathrm{CD}_{38}{ }^{++}$relative to control PBMC cultures $(n=8)$. (B) Percentage of AAV2-specific ASCs in PBMCs from seropositive versus seronegative donors. (C) Percentage of AAV2-specific TNF- $\alpha^{+} C D 8^{+}$T cells in PBMCs comprising or not capsid-specific ASCs. (D) Percentage of AAV2-specific IFN- $\gamma^{+}$NK cells in PBMCs comprising or not capsid-specific ASCs. (E) Concentration of anti-AAV2 IgM secreted in PBMC cultures obtained from AAV2-seropositive donors. (F) Percentage of B cell differentiation in PBMCs obtained from AAV2-seropositive donors, stimulated with an antigen pool of peptides and supplemented with cytokine-neutralizing antibodies or an isotype control ( \pm SD). The numbers of ASCs in cultures with the isotype control were considered to be $100 \%$ of the antigen-specific B cell differentiation $(n=4)$. $\alpha$-IL-1 $\beta$, anti-IL-1 $\beta ; \alpha-I L-6$, anti-IL-6. (G) Concentration of anti-AAV2 IgM secreted in PBMC cultures, stimulated with the AAV2 capsid particles and supplemented with cytokine-neutralizing antibodies or an isotype control. PBMCs were obtained from AAV2-seropositive donors $(n=3)$. (H) Experimental design for the data shown in I and J. On days -2, -1, 0, 1, 2, and 7, mice were injected i.p. with $1 \mathrm{mg} / \mathrm{kg}$ anti-IL-1 $\beta$ - or anti-IL-6-neutralizing antibodies or the corresponding isotype control (Ab). All mice received AAV8-hF.IX by i.v. injection (10 ${ }^{9} \mathrm{vg}$ ) on day 0 . Blood was collected on day 21. (I) Effect of anti-IL-1 $\beta$ - and anti-IL-6-neutralizing antibodies on the anti-AAV8 antibody titers in mouse blood ( $n=5 / g r o u p)$. (J) VGCN per cell, measured by qPCR in mouse liver 3 months after vector injection. The box plots in B, C, D, I, and J show the median \pm SD. ${ }^{*} P<0.05$ and ${ }^{* * *} P<0.001$, by nonparametric Mann-Whitney $U$ test $(\mathbf{A}-\mathbf{D}), 2$-tailed Student's $t$ test (E), and nonparametric Kruskal-Wallis 1-way ANOVA with Dunn's multiple comparisons test (F, G, I, and J).

\section{Discussion}

Despite the clinical success achieved with AAV vectors in several recent gene transfer trials $(1-4,23,24)$, the variability of outcomes across trials and across subjects within the same trials indicates that not all elements affecting immune responses to the AAV vectors are well understood (7). In the current study, using CyTOF and conventional flow cytometry, we characterized immune responses to the AAV capsid in a cohort of healthy individuals. Except for reports of activation of $\mathrm{T}$ cells $(6,22)$ and nonparenchymal liver cells (13), relatively little is known about the mechanisms underlying cellular and humoral immune responses to the AAV capsid in humans. Key questions are how to predict the risk of cytotoxic reactions against transduced cells and how to control both $\mathrm{T}$ and
B cell responses triggered by AAV vector administration. Aiming at this goal, we analyzed 14 cell subsets present in human blood and identified previously unknown capsid-responding cell populations, i.e., NK cells and moDCs.

Our results demonstrated that the main signature of the immune response to the AAV capsid in the blood of healthy individuals is characterized by the secretion of IL- $1 \beta$ and IL- 6 cytokines by moDCs, as observed in $72 \%$ of donors. Production of these cytokines was triggered by both AAV-derived peptides and whole capsid, which is in agreement with published work showing that complex antigens, but also peptides, can activate TLRs (51). As expected for an innate immune response, it occurred irrespective of the donors' AAV serological status. It has been shown 
in mice that several types of DCs collaborate to cross-prime capsid-specific CD8 ${ }^{+} \mathrm{T}$ cells (15). An open question remains as to whether moDCs could also participate in this process. Since the expression of CD86 enables specifically naive $\mathrm{T}$ cell activation, its absence on moDCs exposed to AAV could partially explain the relatively low immunogenicity of the AAV capsid. Results similar to ours were obtained when monocytes differentiated in vitro into DCs were transduced with recombinant AAV-1 (rAAV-1) or rAAV-2, and CD86 expression remained unchanged (52). Whether AAV-induced activation of moDCs depends on TLR2 and CD14 molecules and whether this is important for capsid-specific $\mathrm{T}$ cell priming will be the subjects of further studies. Likewise, the mechanism underlying moDC activation and cytokine production and whether activation results from direct interaction with AAV peptides or via interaction with other APCs remain to be elucidated.

Further, using high-dimensional CyTOF analysis and conventional flow cytometry, we demonstrated that PBMCs isolated from AAV-seronegative donors contained capsid-specific IFN- $\gamma^{+}$ CD16 ${ }^{\text {bright }}$ CD56 ${ }^{\text {dim }}$ NK cells. We have also shown that all AAV serotypes (AAV-2, -5, and -8) tested, but no other viral antigens (flu, $\mathrm{EBV}$, or $\mathrm{CMV}$ ), triggered the secretion of IFN- $\gamma$ in this cell population. Recent findings on the role of antigen-specific memory NK cells in viral immunity are in agreement with the results presented here $(53,54)$. Since CD $16^{\text {bright }}$ CD $56^{\text {dim }}$ NK cells are known to express high levels of killer Ig-like receptors (KIRs), which enable them to recognize particular viral peptides (55-58), it is possible that a discrete clonal population of NK cells, able to recognize AAV, may circulate in human blood. More studies will be needed to better define the role of NK cells in anti-capsid immunity and to understand the relationship between prior exposure to $\mathrm{AAV}$ and the activation state of this cell population. As suggested by the unchanged levels of granzyme B and the degranulation marker CD107a, capsid-specific NKs were not cytotoxic, and cytokine secretion in response to the AAV capsid antigen was transient. Thus, it is difficult to speculate whether these cells may become cytotoxic in the context of AAV gene therapy and as such participate in the clearance of AAV-transduced cells. Nevertheless, although preliminary, our results in NK cells suggest that it may be informative to include NK-specific panels in immunomonitoring protocols used for the follow-up of subjects undergoing AAV gene transfer.

Previous studies of T cell responses to the AAV capsid have been mainly focused on measuring IFN- $\gamma$ secretion. In these studies, capsid-specific IFN- $\gamma^{+} \mathrm{CD} 8^{+} \mathrm{T}$ cells could be detected only in a small subset of individuals, although the frequency of detection was considerably increased after long-term cell restimulation in vitro $(\sim 30$ days) $(22,25,27)$. Since prolonged culture in vitro modifies the cell phenotype, one limitation of these approaches is that they are unlikely to reflect genuine characteristics of capsid-specific T cells circulating in the blood. This may also explain why studies published to date have failed to show a clear correlation between the detection of IFN- $\gamma^{+}$capsid-specific $\mathrm{CD} 8^{+} \mathrm{T}$ cells and AAV seropositivity in healthy donors $(22,26,27)$. Conversely, they may reflect the setting of gene transfer in vivo, where immune cells are exposed to important doses of capsid antigen, an exposure that persists for several weeks and results in the detection of IFN- $\gamma^{+} \mathrm{CD}^{+} \mathrm{T}$ cells $(6,22,59)$.

Our study differs substantially from previous reports and adds critical insights into the interactions between AAV and the host immune system. Here, we provide evidence that capsid-specific memory $\mathrm{CD}^{+} \mathrm{T}$ cells can be readily detected ex vivo by tracking TNF- $\alpha$ secretion. In contrast with previous reports $(22,25,26)$, these cells could only be detected in AAV-seropositive individuals. To exclude the possibility that IFN- $\gamma$ expression was below the limit of detection in this rare antigen-specific $\mathrm{T}$ cell population, we expanded AAV capsid-specific T cells in vitro for 12 days using empty AAV particles or the HLA- $B^{*} 0702$-restricted VP1 ${ }_{372-380}$ epitope $(6,22)$. While the frequency of TNF- $\alpha^{+}$capsid-specific $C D 8^{+} \mathrm{T}$ cells increased by approximately 8 -fold after the expansion, IFN- $\gamma$ secretion was not efficiently detected. These results have clear implications for immunomonitoring performed in the context of AAV gene therapy trials, as they underscore the potential limitation of the IFN- $\gamma$ ELISpot assay currently used to detect cytotoxic responses. They may also explain why in some trials elevation of liver enzymes was detected following gene transfer in the absence of IFN- $\gamma$ detection in PBMCs (3). Our study supports this hypothesis, as it shows that $\mathrm{CD} 8^{+} \mathrm{T}$ cells restimulated with the $\mathrm{VP} 1_{372-380}$ epitope (22) secrete cytotoxic granules (CD107a) in the presence of target cells, without concomitant IFN- $\gamma$ secretion. Thus, to gain a better understanding of the mechanisms driving the immunemediated toxicities observed in AAV gene therapy trials, current immunomonitoring protocols based on IFN- $\gamma$ ELISpot should be supplemented with direct measurements of cytotoxicity, such as perforin or granzyme secretion. Additionally, as suggested by the CyTOF profiling of AAV immune responses in healthy donors, ideally, markers of $\mathrm{T}$ cell activation and function should be measured along with the expression of CD57 and PD-1 to provide a more comprehensive characterization of cell-mediated immunity to AAV.

Humoral responses to the AAV capsid remain an important obstacle to the widespread application of AAV-mediated gene therapy and limit the ability to re-dose the vector when needed (60). Transient depletion of B cells and T cells has been shown to at least partially address the issue (61-64). Yet, global immunosuppression is not without risks for patients, as it increases the susceptibility to infections, leads to reactivation of viruses (65), and can inhibit Tregs that provide transgene tolerance following AAV gene transfer (66). Therefore, targeted regimens that could be applied during gene therapy and would selectively target antigen-specific B cells without inducing profound suppression are needed. To this aim, our findings uncover a previously unknown role of IL-1 $\beta$ and IL- 6 in anti-capsid antibody formation, potentially providing a new approach to curtail humoral immune responses to AAV vectors with direct clinical application. This is in agreement with recent work in mice highlighting the central role of innate immunity in shaping adaptive immune responses to both the capsid (15) and the vector-encoded transgene (67). Indeed, our results demonstrate that neutralization of IL-1 $\beta$ and IL- 6 in PBMC cultures in vitro does not affect flu-specific ASCs, whereas it greatly diminishes the frequency of AAV-specific ASCs and anti-AAV2 antibody levels. While additional studies are needed to understand the mechanism(s) underlying these findings, the approach to anti-AAV blockade presented here offers the advantage of not requiring complete B cell depletion (64). Accordingly, clinical trials of IL-1 $\beta$-neutralizing antibodies showed excellent safety and no significant increase of infection rates $(68,69)$. IL-1 $\beta$ neutralizing antibodies were also efficient in our mouse model of 
AAV8-based gene transfer, which further supports the potential efficacy of this approach in vivo. Interestingly, IL-6-neutralizing antibodies reduced capsid-specific ASCs and antibody levels only in assays performed with human cells. Several reasons may explain this observation and are mainly related to the fact that the human and mouse immune systems differ in multiple aspects (70). Unlike humans, mice are not natural hosts for AAV, although they readily develop anti-AAV antibodies upon AAV vector injection (31). Additionally, in mice, the TLR2 expression pattern and regulation are different (70) and, unlike in humans (13), anti-AAV antibody formation in mice is TLR2 independent (21).

In recent years, the field of gene transfer coalesced around the development of new recombinant capsids permitting more efficient transduction, specific tissue targeting, or escape from preexisting antibodies (71-74). The immunogenicity of these newly developed capsids remains an open question. While animal models do not accurately reflect the immune response to the AAV capsid $(17,28,29)$, the in vitro assays described in this report may be easily adapted to develop a tool to study and predict the immunogenicity of recombinant AAV capsid particles. Additionally, measurement of the release of cytokines such as IL-1 $\beta$ and IL- 6 in immune cells may provide insight into innate immune responses to AAV vectors in humans undergoing gene transfer.

Finally, in our study we associated the immune readouts with the AAV serology of donors. This approach highlighted the existence of 2 distinct profiles of host immune responses to the AAV capsid. The first profile, observed only in seronegative individuals, is associated with the transient activation of a discreet subset of $\mathrm{CD} 16^{\text {bright }} \mathrm{CD} 56^{\mathrm{dim}} \mathrm{NK}$ cells. Indeed, these cells are prominent innate immune effectors known to rapidly respond to infection without prior sensitization and are activated by target cells rather than by the sole presence of cytokines (75). The second profile, observed only in seropositive and hence "AAV-experienced" individuals, is based on the presence of capsid-specific memory CD ${ }^{+}$ T cells and ASCs.

Overall, this study provides a comprehensive description of human immune responses against the AAV capsid, points to the use of markers of immune system activation that should be monitored in AAV clinical trials, and describes a new immunomodulatory strategy to control host humoral responses against AAV vectors in gene therapy.

\section{Methods}

Human primary cells and cell lines. Cryopreserved PBMCs were purchased from Cellular Technology Limited (CTL), and buffy coats were purchased from Etablissement Français du Sang (EFS). PBMCs were isolated with Ficoll-Hypaque gradient centrifugation according to standard protocols and cryopreserved in liquid nitrogen vapor until assayed. In total, 44 normal donors were analyzed. The average donor was 33 years of age ( \pm 14 years; range: $18-65$ years). The average age of donors in the AAV2-seropositive group tended to be higher than those in the AAV2-seronegative group, but the difference was not statistically significant (AAV2-seropositive, $40 \pm 16$ years [ $n=13]$ vs. AAV2 seronegative, $30 \pm 14$ years $[n=12]$ ).

The HLA- $B^{*} 0702$ cell line (ATCC-CRL-2371) purchased from LGC Standards and pulsed with appropriate peptides was used as APCs for expanded antigen-specific $\mathrm{CD} 8^{+} \mathrm{T}$ cells.
$A A V$ vectors and antigens. The AAV8 vector carrying the cDNA encoding for human factor IX (hF.IX) under control of a liver-specific promoter (AAV8-hAAT-F.IX) (48) and empty-AAV capsids were produced using a slight modification of the adenovirus-free transient transfection method previously described $(76,77)$. Briefly, HEK293 cells were transfected and harvested 72 hours thereafter. After sonication and benzonase treatment, vectors were purified using 2 successive ultracentrifugation rounds in cesium chloride density gradients. The AAV8-hAAT-F.IX genome was quantified using quantitative realtime PCR (qPCR) (48). Empty AAV capsids were used for PBMCs stimulation at a concentration of $10 \mu \mathrm{g} / \mathrm{ml}$.

Peptide pools consisted of 15-mer sequences with 11 amino acids overlap, covering the complete sequence of a chosen viral protein. Human flu NP protein (Miltenyi Biotec), EBV BMLF-1 protein (Miltenyi Biotec), VP1 capsid protein from AAV2, AAV8 (ChinaPeptides) or AAV5 (Mimotopes) were used at $1 \mu \mathrm{g}$ of each peptide $/ \mathrm{ml}$. As a positive control, a pool of $23 \mathrm{MHC}$ class I-restricted viral peptides from human cytomegalovirus, EBV and flu (CEF) was used at $2 \mu \mathrm{g}$ of each peptide/ml (Mabtech).

The HLA- $B^{*} 0702$-restricted peptides AAV2 VP1 $1_{372-380}$ (VPQYGYLTL) or control peptide EBV EBNA $3 A_{247-255}$ (RPPIFIRRL) were purchased from ChinaPeptides and used at $10 \mu \mathrm{M}$ concentration. For unspecific stimulation PMA and ionomycin (used at $50 \mathrm{ng} / \mathrm{ml}$ and $1 \mu \mathrm{g} / \mathrm{ml}$, respectively) or $5 \mu \mathrm{g} / \mathrm{ml}$ LPS were used (Sigma-Aldrich).

Cell culture. PBMCs were maintained in AIM-V Glutamax medium (Gibco, Thermo Fisher Scientific) for 6 hours (T cell assays), 24 hours, and 48 hours (NK and DC assays), or for 7 days (B cell assays).

$T$ cell expansion. Antigen-specific $\mathrm{CD} 8^{+} \mathrm{T}$ cell expansion was performed as described previously (78). Briefly, PBMCs were stimulated with AAV2 or EBV HLA- $B^{*} 0702$-restricted peptides on day 0 in AIM V medium (Gibco, Thermo Fisher Scientific) supplemented with 1,000 $\mathrm{U} / \mathrm{ml}$ granulocyte-macrophage-CSF (GM-CSF) and $500 \mathrm{U} / \mathrm{ml} \mathrm{IL}-4$. After 24 hours (day 1), a maturation cocktail was added (1,000 U/ml TNF- $\alpha, 10 \mathrm{ng} / \mathrm{ml} \mathrm{IL-1 \beta ,} 1 \mu \mathrm{M}$ PGE2, $0.5 \mathrm{ng} / \mathrm{ml} \mathrm{IL}-7)$, and then cells were maintained in RPMI medium (Gibco, Thermo Fisher Scientific) supplemented with $10 \% \mathrm{FBS}, 25 \mathrm{ng} / \mathrm{ml} \mathrm{IL-15,} 100 \mathrm{U} / \mathrm{ml} \mathrm{IL-2}$, and $5 \mathrm{ng}$ / $\mathrm{ml}$ IL-7 for 2 weeks.

CyTOF mass cytometry. PBMCs obtained from 4 healthy donors were stained and analyzed 48 hours after restimulation with empty AAV2 capsid particles or 6 hours after stimulation with PMA and ionomycin. Cytokine secretion in cell cultures was blocked by the addition of GolgiPlug/GolgiStop (BD) for 5 hours prior to cell harvesting and staining. The Maxpar Cytoplasmic/Secreted Antigen Staining Protocol (PRD017 V2 02/14, Fluidigm) was used, with the only modification being that for the cellular permeabilization and intracellular staining, the Cytofix/Cytoperm Kit from BD was used. Staining antibodies were validated by the manufacturer DVS Sciences. Unless otherwise indicated, all antibodies for CyTOF were purchased from DVS Sciences. The cell surface was stained with the following antibodies: CD3 (catalog 3154003C); CD4 (catalog 3145001C); CD8a (catalog 3146001C); CD16 (catalog 3148004C); CD19 (catalog 3142001C); CD25 (catalog 3169003C); CD27 (catalog 3162009C); CD38 (catalog 3167001C); CD45 (catalog 3141009C); CD45RA (catalog 3153001C); CD45RO (catalog 3165011C); CD57 (catalog 3172009C); and HLA-DR (catalog 3174001C). Then, intracellular staining was performed with anti-granzyme B (catalog 3171002C); IFN- $\gamma$ (catalog 3168005C); IL-2 (cata$\log 3158007 \mathrm{C})$; IL-5 (catalog 3143003C); IL-10 (catalog 3166008C); 
IL-17A (catalog 3164002C); and TNF- $\alpha$ (catalog 3152002C). FoxP3 was detected by PE-conjugated anti-FoxP3 antibody (catalog 320208, BioLegend) and a secondary anti-PE antibody (catalog 3156005B, DVS Sciences). Data were collected on a CyTOF2 Mass Cytometer (Fluidigm software, version 6.0.626) with dual-count calibration, noise reduction, a cell length threshold between 10 and 150 pushes, and a lower convolution threshold equal to 10 . The resulting flow cytometry standard (fcs) files were normalized with the MatLab Compiler normalizer using a signal from 4-Element EQ Beads (Fluidigm). The fcs files were analyzed using FlowJo software (Tree Star).

ICS assay and flow cytometry. PBMCs were seeded at 2.5 or $5 \times$ $10^{6}$ cells per well. When pertinent, cytokine secretion in cell cultures was blocked by the addition of GolgiPlug/GolgiStop (BD) for 5 hours, and anti-human CD107a (328624, BioLegend) was added at this step according to the manufacturer's protocol. Prior to cell staining, the FcR binding inhibitor (eBioscience) was used. Dead cells were stained with the Zombie Yellow Fixable Viability Kit (BioLegend) or the LIVE/ DEAD Fixable Aqua Dead Cell Stain Kit (Life Technologies, Thermo Fisher Scientific). Cell-surface staining was performed with the following anti-human antibodies: CD4 (catalog 317414) and CD16 (catalog 302028) from BioLegend; CD3 (catalog A07748), CD8 (catalog B00067), CD19 (catalog IM2708U), and CD56 (catalog B46024) from Beckman Coulter; HLA-DR (catalog 560743), IgG (catalog 564229), IgD (catalog 561302), CD14 (catalog 563698), CD19 (catalog 555412), CD24 (catalog 562788), CD25 (catalog 564034), CD27 (catalog 555441), CD38 (catalog 560677), CD80 (catalog 555683), and CD86 (catalog 561129) from BD Biosciences; and CD11c (catalog 12-011642) from eBioscience. Prior to intracellular staining, cells were fixed with the BD Cytofix/Cytoperm Kit for 30 minutes and stained with the following antibodies as needed: IFN- $\gamma$ (catalogs 45-7319-42 and 12-7319-82) and IL-6 (catalog 17-7069-42) from eBioscience; and/or granzyme B (catalog 515408), IFN- $\gamma$ (catalog 502506), IL-1 $\beta$ (catalog 511710), IL-2 (catalog 500310), and TNF- $\alpha$ (catalog 502909) from BioLegend. Data were collected on a Cytoflex S (Beckman Coulter) or a FACSCanto II (BD) and analyzed with FlowJo software (Tree Star).

Quantification of antibodies in plasma or culture supernatants. Anti-AAV antibody titers were measured by standard ELISA using 96-well Nunc MaxiSorp Immunoplates (Dutscher) coated overnight with AAV2 or AAV8 in a carbonate buffer at a final concentration of $10 \mu \mathrm{g} / \mathrm{ml}$. Plates were blocked with PBS containing $2 \%$ BSA. Antihuman or anti-mouse IgG antibodies conjugated with HRP (Southern Biotech) were used. The enzymatic reaction was developed with 3,3',5,5'-tetramethylbenzidine (TMB) (BD) stopped with $\mathrm{H}_{2} \mathrm{SO}_{4} 3 \mathrm{M}$ solution. OD was measured at $450 \mathrm{~nm}$ using an ENSPIRE Microplate Reader (PerkinElmer). Sera from healthy donors were considered seropositive when titers were higher than 1:3.

Human anti-AAV2 IgM measurements were performed in conditioned medium 7 days after stimulation of PBMCs with AAV2 or flu pools of peptides and compared with the level of nonstimulated cultures. Human biotin-conjugated anti-IgM antibodies (Sigma-Aldrich) were used, and the enzymatic reaction was developed with Streptavidin Alkaline Phosphatase and Alkaline Phosphatase Yellow (pNPP) Liquid Substrate (Sigma-Aldrich). OD was measured at $405 \mathrm{~nm}$ using a Spark Microplate Reader (Tecan).

Anti-flu antibodies in plasma from healthy donors were measured using an Anti-Influenza A IgG Human ELISA Kit (Abcam) according to the manufacturer's protocol. Samples were considered positive if the absorbance value was more than $10 \%$ over the cutoff value of the control $\left(\mathrm{OD}_{450 \mathrm{~nm}}=1\right)$ provided with the kit.

Cytokine multiplex assays. Supernatants from 24-hour PBMC cultures, stimulated with the pools of AAV2 or flu peptides, were analyzed on a Luminex platform (Bio-Plex 200, Bio-Rad) using the Pro-human Cytokine 17-Plex Immunoassay (M5000031YV, Bio-Rad) according to the manufacturer's instructions.

Autologous DC preparation and recall. On day 10 of the antigen-specific $\mathrm{T}$ cell expansion in vitro, PBMCs from the same donor were thawed, and plastic-adherent cells were stimulated with the indicated antigens (Figure 3B) in AIM V medium (Gibco, Thermo Fisher Scientific) supplemented with $1,000 \mathrm{U} / \mathrm{ml} \mathrm{GM-CSF}$ and $500 \mathrm{U} / \mathrm{ml}$ IL-4. On day 12 , maturation cocktail was added (1,000 U/ml TNF- $\alpha$, $10 \mathrm{ng} / \mathrm{ml} \mathrm{IL-1 \beta ,} 1 \mu \mathrm{M}$ PGE2, $0.5 \mathrm{ng} / \mathrm{ml} \mathrm{IL-7)}$ to the wells. Twenty-four hours later, the antigen-loaded DCs were washed with PBS and covered with the suspension of expanded T cells.

HLA DMr assays. Prior to staining, PBMCs were incubated in PBS with $50 \mathrm{nM}$ reversible protein kinase inhibitor (PKI) and dasatinib (Clinisciences) (79). PE-labeled HLA-B ${ }^{\star} 0702$ DMrs loaded with $\mathrm{VP1} 1_{372-380}$ or control peptide EBNA $3 \mathrm{~A}_{247-255}$ were used according to the manufacturer's instructions (Immudex), and then the ICS assay was performed as described above.

ELISpot assay. Dual-color FluoroSpot was performed according to the manufacturer's protocol (CTL Inc.) and a previously described protocol (23). Briefly, PBMCs were seeded in triplicate $\left(3 \times 10^{5}\right.$ cells/ well) in AIM V Glutamax medium (Gibco, Thermo Fisher Scientific) into an IFN- $\gamma$ - and TNF- $\alpha$-coated, 96-well ELISpot precoated plate and stimulated with the AAV2 pool of peptides (145 peptides pooled, final concentration $1 \mu \mathrm{g} / \mathrm{ml} /$ peptide), CEF (32 peptides pooled, final concentration $1 \mu \mathrm{g} / \mathrm{ml} /$ peptide), or PMA and ionomycin. Twenty-four hours later, the plates were washed with $0.05 \%$ Tween-20 in PBS, and tertiary antibodies were added and incubated for 2 hours at room temperature. Plates were then washed again with $0.05 \%$ Tween-PBS and air-dried. Fluorescent spots were counted using a CTL FluoroSpot Reader. Results are expressed as spot-forming units (SFU) $/ 10^{6}$ cells. A response was considered positive for SFU of 50 or higher and at least 3 times higher than SFU levels in the nonstimulated (No Ag) control.

In vitro blockade of IL- $1 \beta$ and IL- 6 cytokines. Human PBMCs stimulated with the AAV2 or flu pools of peptides were cultivated for 7 days in medium supplemented with IL-6 antibody (catalog MAB206-100), IL-1 $\beta$ antibody (MAB601-100), or IgG1 isotype control (MAB002) from R\&D Systems, at a final concentration of $7.5 \mu \mathrm{g} / \mathrm{ml}$.

Blockade of $I L-1 \beta$ and IL- 6 cytokines in vivo. Six-week-old male C57BL/6J mice were purchased from Charles River Laboratories. AAV8-hAAT-F.IX vector $\left(1 \times 10^{9} \mathrm{vg}\right)$ was administered via the tail vein of all animals on day 0 . On days $-2,-1,0,1,2$, and 7, mice were injected i.p. with $1 \mathrm{mg} / \mathrm{kg}$ anti-IL-1 $\beta$-neutralizing antibody (BE0246), antiIL-6-neutralizing antibody (BE0046), or the corresponding isotype IgG control (BE0290 or BE0091) from BioXcell (5 mice/group). Blood was collected on day 21 and livers 3 months after vector injection. C57BL/6 mice were obtained from The Jackson Laboratory.

Viral VGCN analysis. Total DNA was extracted from $100 \mathrm{mg}$ frozen liver tissue using the MagNA Pure 96 DNA and Viral DNA Small Volume Kit (Roche Diagnosis) according to the manufacturer's instructions. VGCNs measured by quantitative PCR (qPCR) were normalized by the copies of the titin gene measured in each sample. qPCR was performed on an ABI PRISM 7900 HT Sequence Detector (Agilent Technologies) 
using Absolute ROX Mix (Thermo Fisher Scientific) and the following specific primers for the vector genome: forward, 5'-GCCACTAAGGATTCTGCAGT-3', reverse, 5'-CTGCACTTACCGAAAGGAGT-3'; for mouse titin (mTTN): forward, 5'-AAAACGAGCAGTGACGTGAGC-3' , reverse, 5' -TTCAGTCATGCTGCTAGCGC-3'.

Statistics. Statistical analyses were performed using GraphPad Prism, version 7.0 (GraphPad Software). Normal distribution of the samples was tested using the D'Agostino-Pearson normality test. All data shown were analyzed by nonparametric tests, with the exception of the FluoroSpot data in Figure 4D (80) and the data in Figure 6E, which were analyzed with a Student's $t$ test. A Mann-Whitney $U$ test was used for the comparison of data sets composed of 2 groups, except in Figure 3B, in which a Wilcoxon signed-rank test was used because of the absence of variance in the control group. A nonparametric KruskalWallis 1-way ANOVA with Dunn's multiple comparisons test was used for analysis of all the data sets containing more than 2 groups. $P$ values of less than 0.05 were considered statistically significant.

Study approval. All blood samples were obtained from commercial providers with written informed consent from the donor and were used in accordance with Declaration of Helsinki principles. Animal studies were performed in accordance with the current European legislation on animal care and experimentation (2010/63/EU) and approved by the institutional ethics committee of the Centre d'Exploration et de Recherche Fonctionnelle Expérimentale in Evry, France (protocol APAFIS 3055-20151019213299180).

\section{Author contributions}

$\mathrm{KK}$ and FM designed experiments and wrote the manuscript. KK, PJA, CL, RH, SM, HCV, and PV performed experiments. FC provided reagents. PV helped with manuscript preparation. KK and GR analyzed data. FM supervised the project.

\section{Acknowledgments}

We gratefully acknowledge Victor Appay (CIMI/Inserm U1135) for helpful scientific discussions and the staff of the CyTOF technical platform Plateforme de Cytométrie de la Pitié-Salpetrière (CyPS) (Paris, France), and Aurélien Corneau and Catherine Blanc (both from CyPS) for their technical help and analytic support. This work was supported by the European Union: an FP7PEOPLE-2012-CIG Career Integration Grant (grant agreement 333628, NosMod, to FM); an ERC-2013-CoG Consolidator Grant (grant agreement 617432, MoMAAV, to FM); a European Union Research and Innovation Program grant (agreement 667751, Myocure, to FM); an E-Rare2 grant SMART-HaemoCare grant (to FM). This work was also supported by a Bayer Early Career Investigator Award (to FM).

Address correspondence to: Federico Mingozzi, Immunology and Liver Gene Therapy, INSERM S951, 1 rue de l'Internationale, 91000 Evry, France. Phone: 33.0.169.47.28.28; Email: fmingozzi@genethon.fr.
1. George LA, et al. Hemophilia B gene therapy with a high-specific-activity factor IX variant. $\mathrm{NEnglJ}$ Med. 2017;377(23):2215-2227.

2. Mendell JR, et al. Single-fose hene-teplacement therapy for spinal muscular atrophy. N EnglJ Med. 2017;377(18):1713-1722.

3. Rangarajan S, et al. AAV5-factor VIII gene transfer in severe hemophilia A. N Engl J Med. 2017;377(26):2519-2530.

4. Miesbach W, et al. Gene therapy with adenoassociated virus vector 5-human factor IX in adults with hemophilia B. Blood. 2018;131(9):1022-1031.

5. Russell S, et al. Efficacy and safety of voretigene neparvovec (AAV2-hRPE65v2) in patients with RPE65-mediated inherited retinal dystrophy: a randomised, controlled, open-label, phase 3 trial. Lancet . 2017;390(10097):849-860.

6. Manno CS, et al. Successful transduction of liver in hemophilia by AAV-factor IX and limitations imposed by the host immune response. Nat Med. 2006;12(3):342-347.

7. Ertl HCJ, High KA. Impact of AAV capsidspecific T-cell responses on design and outcome of clinical gene transfer trials with recombinant adeno-associated viral vectors: an evolving controversy. Hum Gene Ther. 2017;28(4):328-337.

8. Mingozzi F, High KA. Overcoming the host immune response to adeno-associated virus gene delivery vectors: the race between clearance, tolerance, neutralization, and escape. Annu Rev Virol. 2017;4(1):511-534.

9. Zhu J, Huang X, Yang Y. The TLR9-MyD88 pathway is critical for adaptive immune responses to adeno-associated virus gene therapy vectors in mice. J Clin Invest. 2009;119(8):2388-2398.

10. Zaiss AK, Liu Q, Bowen GP, Wong NC, Bart- lett JS, Muruve DA. Differential activation of innate immune responses by adenovirus and adeno-associated virus vectors. J Virol. 2002;76(9):4580-4590.

11. Vandendriessche T, et al. Efficacy and safety of adeno-associated viral vectors based on serotype 8 and 9 vs. lentiviral vectors for hemophilia B gene therapy. J Thromb Haemost. 2007;5(1):16-24.

12. Suzuki M, et al. Differential type I interferondependent transgene silencing of helper-dependent adenoviral vs. adeno-associated viral vectors in vivo. Mol Ther. 2013;21(4):796-805.

13. Hosel M, et al. Toll-like receptor 2-mediated innate immune response in human nonparenchymal liver cells toward adeno-associated viral vectors. Hepatology. 2012;55(1):287-297.

14. Manukyan M, et al. Binding of lipopeptide to CD14 induces physical proximity of CD14, TLR2 and TLR1. Eur JImmunol. 2005;35(3):911-921.

15. Rogers GL, et al. Plasmacytoid and conventional dendritic cells cooperate in crosspriming AAV capsid-specific CD8(+) T cells. Blood. 2017;129(24):3184-3195.

16. $\mathrm{Li} \mathrm{H}$, et al. Adeno-associated virus vectors serotype 2 induce prolonged proliferation of capsid-specific CD8+ T cells in mice. Mol Ther. 2011;19(3):536-546.

17. Li H, et al. Pre-existing AAV capsid-specific CD8 ${ }^{+}$ $\mathrm{T}$ cells are unable to eliminate AAV-transduced hepatocytes. Mol Ther. 2007;15(4):792-800.

18. Gil-Farina I, et al. IL12-mediated liver inflammation reduces the formation of AAV transcriptionally active forms but has no effect over preexisting AAV transgene expression. PLoS One. 2013;8(7):e67748.

19. Martino AT, et al. Engineered AAV vector minimizes in vivo targeting of transduced hepato- cytes by capsid-specific CD $8^{+} \mathrm{T}$ cells. Blood. 2013;121(12):2224-2233.

20. Mingozzi F, High KA. Therapeutic in vivo gene transfer for genetic disease using AAV: progress and challenges. Nat Rev Genet. 2011;12(5):341-355.

21. Rogers GL, et al. Unique roles of TLR9- and MyD88-dependent and -independent pathways in adaptive immune responses to AAV-mediated gene transfer. JInnate Immun. 2015;7(3):302-314.

22. Mingozzi F, et al. CD8(+) T-cell responses to adeno-associated virus capsid in humans. Nat Med. 2007;13(4):419-422.

23. Nathwani AC, et al. Long-term safety and efficacy of factor IX gene therapy in hemophilia B. N Engl JMed. 2014;371(21):1994-2004.

24. Nathwani AC, et al. Adenovirus-associated virus vector-mediated gene transfer in hemophilia B. NEnglJMed. 2011;365(25):2357-2365.

25. Hui DJ, et al. AAV capsid CD8 ${ }^{+} \mathrm{T}$-cell epitopes are highly conserved across AAV serotypes. Mol Ther Methods Clin Dev. 2015;2:15029.

26. Veron $P$, et al. Humoral and cellular capsid-specific immune responses to adeno-associated virus type 1 in randomized healthy donors. JImmunol. 2012;188(12):6418-6424.

27. Vandamme C, et al. ASGCT meeting. Session on immunological aspects of gene therapy II: AAV vectors, 697. 2016. http://www.abstractsonline. com/pp8/\#!/4077/presentation/340.

28. Li C, et al. Adeno-associated virus type 2 (AAV2) capsid-specific cytotoxic T lymphocytes eliminate only vector-transduced cells coexpressing the AAV2 capsid in vivo. JVirol. 2007;81(14):7540-7547.

29. Wang L, Figueredo J, Calcedo R, Lin J, Wilson JM. Cross-presentation of adeno-associated virus 
serotype 2 capsids activates cytotoxic $\mathrm{T}$ cells but does not render hepatocytes effective cytolytic targets. Hum Gene Ther. 2007;18(3):185-194.

30. Sudres M, et al. MyD88 signaling in B cells regulates the production of Th1-dependent antibodies to AAV. Mol Ther. 2012;20(8):1571-1581.

31. Chirmule N, et al. Humoral immunity to adenoassociated virus type 2 vectors following administration to murine and nonhuman primate muscle. JVirol. 2000;74(5):2420-2425.

32. Scallan CD, et al. Human immunoglobulin inhibits liver transduction by AAV vectors at low AAV2 neutralizing titers in SCID mice. Blood. 2006;107(5):1810-1817.

33. Calcedo R, Wilson JM. AAV natural infection induces broad cross-neutralizing antibody responses to multiple AAV serotypes in chimpanzees. Hum Gene Ther Clin Dev. 2016;27(2):79-82.

34. Nathwani AC, et al. Safe and efficient transduction of the liver after peripheral vein infusion of self-complementary AAV vector results in stable therapeutic expression of human FIX in nonhuman primates. Blood. 2007;109(4):1414-1421.

35 . Flotte TR, et al. Phase 2 clinical trial of a recombinant adeno-associated viral vector expressing alpha1-antitrypsin: interim results. Hum Gene Ther. 2011;22(10):1239-1247.

36. Jiang $\mathrm{H}$, et al. Effects of transient immunosuppression on adenoassociated, virus-mediated, liver-directed gene transfer in rhesus macaques and implications for human gene therapy. Blood. 2006;108(10):3321-3328.

37. Murphy SL, Li H, Zhou S, Schlachterman A, High KA. Prolonged susceptibility to antibody-mediated neutralization for adeno-associated vectors targeted to the liver. Mol Ther. 2008;16(1):138-145.

38. Sabroe I, Jones EC, Usher LR, Whyte MK, Dower SK. Toll-like receptor (TLR)2 and TLR4 in human peripheral blood granulocytes: a critical role for monocytes in leukocyte lipopolysaccharide responses. JImmunol. 2002;168(9):4701-4710.

39. Collin M, Bigley V, Haniffa M, Hambleton S. Human dendritic cell deficiency: the missing ID? Nat Rev Immunol. 2011;11(9):575-583.

40. Li H, et al. Capsid-specific T-cell responses to natural infections with adeno-associated viruses in humans differ from those of nonhuman primates. Mol Ther. 2011;19(11):2021-2030.

41. Barber DL, et al. Restoring function in exhausted CD8 T cells during chronic viral infection. Nature. 2006;439(7077):682-687.

42. Spits H, Lanier LL. Natural killer or dendritic: what's in a name? Immunity. 2007;26(1):11-16.

43. Daya S, Berns KI. Gene therapy using adenoassociated virus vectors. Clin Microbiol Rev. 2008;21(4):583-593.

44. Boutin S, et al. Prevalence of serum IgG and neutralizing factors against adeno-associated virus (AAV) types 1, 2, 5, 6, 8, and 9 in the healthy population: implications for gene therapy using AAV vectors. Hum Gene Ther. 2010;21(6):704-712.

45. Maliszewski CR, et al. Cytokine receptors and B cell functions. I. Recombinant soluble receptors specifically inhibit IL-1- and IL-4-induced B cell activities in vitro. J Immunol. 1990;144(8):3028-3033.

46. Nakae S, Asano M, Horai R, Iwakura Y. Interleukin-1 beta, but not interleukin-1 alpha, is required for T-cell-dependent antibody production.
Immunology. 2001;104(4):402-409.

47. Kishimoto T. IL-6: from its discovery to clinical applications. Int Immunol. 2010;22(5):347-352.

48. Meliani A, et al. Enhanced liver gene transfer and evasion of preexisting humoral immunity with exosome-enveloped AAV vectors. Blood Adv. 2017;1(23):2019-2031.

49. Gao GP, Alvira MR, Wang L, Calcedo R, Johnston J, Wilson JM. Novel adeno-associated viruses from rhesus monkeys as vectors for human gene therapy. Proc Natl Acad Sci U S A. 2002;99(18):11854-11859.

50. Lisowski L, et al. Selection and evaluation of clinically relevant $\mathrm{AAV}$ variants in a xenograft liver model. Nature. 2014;506(7488):382-386.

51. Myneni SR, et al. Identification of a unique TLR2-interacting peptide motif in a microbial leucine-rich repeat protein. Biochem Biophys Res Commun. 2012;423(3):577-582.

52. Veron P, Allo V, Riviere C, Bernard J, Douar AM, Masurier C. Major subsets of human dendritic cells are efficiently transduced by self-complementary adeno-associated virus vectors 1 and 2 . J Virol. 2007;81(10):5385-5394.

53. Nabekura T, Lanier LL. Tracking the fate of antigen-specific versus cytokine-activated natural killer cells after cytomegalovirus infection. J Exp Med. 2016;213(12):2745-2758.

54. Reeves RK, et al. Antigen-specific NK cell memory in rhesus macaques. Nat Immunol. 2015;16(9):927-932.

55. Stewart CA, et al. Recognition of peptide-MHC class I complexes by activating killer immunoglobulin-like receptors. Proc Natl Acad Sci U S A. 2005;102(37):13224-13229.

56. Beziat $\mathrm{V}$, et al. CMV drives clonal expansion of $\mathrm{NKG}_{2} \mathrm{C}^{+} \mathrm{NK}$ cells expressing self-specific KIRs in chronic hepatitis patients. Eur J Immunol. 2012;42(2):447-457.

57. Fadda L, et al. Common HIV-1 peptide variants mediate differential binding of KIR3DL1 to HLABw4 molecules. J Virol. 2011;85(12):5970-5974.

58. Naiyer MM, et al. KIR2DS2 recognizes conserved peptides derived from viral helicases in the context of HLA-C. Sci Immunol. 2017;2(15):eaal5296.

59. Mingozzi F, et al. AAV-1-mediated gene transfer to skeletal muscle in humans results in dosedependent activation of capsid-specific T cells. Blood. 2009;114(10):2077-2086.

60. Bortolussi G, et al. Life-long correction of hyperbilirubinemia with a neonatal liver-specific AAV-mediated gene transfer in a lethal mouse model of Crigler-Najjar syndrome. Hum Gene Ther. 2014;25(9):844-855.

61. Monahan PE, et al. Proteasome inhibitors enhance gene delivery by AAV virus vectors expressing large genomes in hemophilia mouse and dog models: a strategy for broad clinical application. Mol Ther. 2010;18(11):1907-1916.

62. Mingozzi F, et al. Pharmacological modulation of humoral immunity in a nonhuman primate model of AAV gene transfer for hemophilia B. Mol Ther. 2012;20(7):1410-1416.

63. Mingozzi F, et al. Prevalence and pharmacological modulation of humoral immunity to AAV vectors in gene transfer to synovial tissue. Gene Ther. 2013;20(4):417-424.

64. Corti M, et al. B-cell depletion is protective against anti-AAV capsid immune response: a human subject case study. Mol Ther Methods Clin Dev. 2014;1:14033.

65. Ardehali H, Volmar K, Roberts C, Forman M, Becker LC. Fatal disseminated adenoviral infection in a renal transplant patient. Transplantation. 2001;71(7):998-999.

66. Mingozzi F, et al. Modulation of tolerance to the transgene product in a nonhuman primate model of AAV-mediated gene transfer to liver. Blood. 2007;110(7):2334-2341.

67. Herzog RW, et al. Regulatory T cells and TLR9 activation shape antibody formation to a secreted transgene product in AAV muscle gene transfer. [published online ahead of print August 1, 2017]. Cell Immunol. https://doi.org/10.1016/j. cellimm.2017.07.012

68. Howard C, et al. Safety and tolerability of canakinumab, an IL-1beta inhibitor, in type 2 diabetes mellitus patients: a pooled analysis of three randomised double-blind studies. Cardiovasc Diabetol. 2014;13:94.

69. Tugal-Tutkun I, Pavesio C, De Cordoue A, Bernard-Poenaru O, Gul A. Use of gevokizumab in patients with Behcet's disease uveitis: an international, randomized, double-masked, placebo-controlled study and open-label extension study. [published online ahead of print January 25, 2018]. Ocul Immunol Inflamm. https://doi.org /10.1080/09273948.2017.1421233.

70. Mestas J, Hughes CC. Of mice and not men: differences between mouse and human immunology. JImmunol. 2004;172(5):2731-2738.

71. Pulicherla N, et al. Engineering liver-detargeted AAV9 vectors for cardiac and musculoskeletal gene transfer. Mol Ther. 2011;19(6):1070-1078.

72. Bowles DE, et al. Phase 1 gene therapy for Duchenne muscular dystrophy using a translational optimized AAV vector. Mol Ther. 2012;20(2):443-455.

73. Tse LV, et al. Structure-guided evolution of antigenically distinct adeno-associated virus variants for immune evasion. Proc Natl Acad Sci U S A. 2017;114(24):E4812-E4821.

74. Grimm D, Buning H. Small but increasingly mighty: latest advances in AAV vector research, design, and evolution. Hum Gene Ther. 2017;28(11):1075-1086.

75. Anfossi N, et al. Human NK cell education by inhibitory receptors for MHC class I. Immunity. 2006;25(2):331-342.

76. Matsushita T, et al. Adeno-associated virus vectors can be efficiently produced without helper virus. Gene Ther. 1998;5(7):938-945.

77. Ayuso E, et al. High AAV vector purity results in serotype- and tissue-independent enhancement of transduction efficiency. Gene Ther. 2010;17(4):503-510.

78. Martinuzzi E, et al. acDCs enhance human antigen-specific T-cell responses. Blood. 2011;118(8):2128-2137.

79. Lissina A, et al. Protein kinase inhibitors substantially improve the physical detection of T-cells with peptide-MHC tetramers. JImmunol Methods. 2009;340(1):11-24.

80. Karulin AY, Caspell R, Dittrich M, Lehmann PV. Normal distribution of $\mathrm{CD} 8^{+} \mathrm{T}$-cell-derived ELISPOT counts within replicates justifies the reliance on parametric statistics for identifying positive responses. Cells. 2015;4(1):96-111. 